\title{
Real-Time Observation of Multiexcitonic States in Ultrafast Singlet Fission Using Coherent 2D Electronic Spectroscopy
}

\author{
Artem A. Bakulin ${ }^{1,2}$, Sarah E. Morgan ${ }^{2}$, Tom B. Kehoe ${ }^{2}$, Mark W.B. Wilson', Alex W. Chin', \\ Donatas Zigmantas ${ }^{4}$, Dassia Egorova ${ }^{* 3}$, Akshay Rao $*^{2}$ \\ ${ }^{I}$ FOM Institute AMOLF, 1098 XG Amsterdam, The Netherlands \\ ${ }^{2}$ Cavendish Laboratory, University of Cambridge, JJ Thomson Avenue, Cambridge CB30HE, UK \\ ${ }^{3}$ Institut für Physikalische Chemie, Christian-Albrechts-Universität zu Kiel, Olshausenstr. 40, D-24098 Kiel, \\ Germany \\ ${ }^{4}$ Department of Chemical Physics, Lund University, P.O. Box 124, 22100 Lund, Sweden \\ *Corresponding authors: \\ degorova@gmail.com, ar525@,cam.ac.uk
}

\begin{abstract}
:
Singlet fission is the spin-allowed conversion of a spin-singlet exciton into a pair of spintriplet excitons residing on neighbouring molecules. To rationalise this phenomenon, a multiexcitonic spin-zero triplet-pair state has been hypothesised as an intermediate in singlet fission. However, the nature of the intermediate states and the underlying mechanism of ultrafast fission have not been elucidated experimentally. Here, we study a series of pentacene derivatives using ultrafast 2D electronic spectroscopy and unravel the origin of the states involved in fission. Our data reveal the crucial role of vibrational degrees of freedom coupled to electronic excitations that facilitate the mixing of multiexcitonic states with singlet excitons. The resulting manifold of vibronic states drives sub-100-fs fission with unity efficiency. Our results provide a framework for understanding singlet fission and show how the formation of vibronic manifolds with a high density of states facilitates fast and efficient electronic processes in molecular systems.
\end{abstract}


Singlet fission (SF) is an exciton multiplication process in organic semiconductors that allows one photogenerated spin-singlet excited state to be converted to two spin-triplet excitons. ${ }^{1}$ The two generated spin-triplet excitons are initially correlated to form an overall spin-singlet state, making SF a spin-allowed process in contrast to intersystem crossing that involves a spin flip. For systems where the energy of the lowest lying singlet exciton $(S)$ is close to double the energy of the triplet state $(T)$, such as pentacene and its derivatives, SF can occur on a sub-100fs timescale with every singlet being converted to two triplets. ${ }^{2}$

SF has attracted great attention lately as it enables photovoltaic devices to overcome thermalisation losses by generating two electron-hole pairs per high-energy photon absorbed, potentially allowing single-junction devices that could beat the Shockley-Queisser limit on power conversion efficiency ${ }^{3}$. The first steps towards this goal have been taken with the demonstration of organic solar cells based on pentacene, that show external quantum efficiencies above $129 \%$, the highest for any solar technology to date. ${ }^{4,5}$

Despite advances in the experimental characterization of SF in several molecular systems ${ }^{6-13}$ as well as extensive theoretical work, ${ }^{1,14-22}$ the fundamental mechanism of ultrafast SF remains unclear. In the kinetic model proposed by Merrifield and co-workers ${ }^{23}$ the process can be represented as

\section{$S \rightarrow T T \rightarrow T+T$}

Where: $S$ is the lowest singlet excited singlet state, $T$ is the molecular triplet state and $T+T$ is a pair of fully independent $T$ states. $T T$ corresponds to a doubly excited pair of spin-correlated triplets, forming an overall spin singlet. The $T T$ state, often referred to as the multiexciton state, is considered a dark state that cannot be optically populated from the ground state $g$, but serves as an intermediate to the formation of free independent triplets $T+T$.

Current theoretical models for SF focus on characterising the electronic structure of the $S$ and $T T$ states, and the interplay between them. ${ }^{1,20,21}$ While it is clear that the coupling between $S$ and the $T T$ state controls the dynamics of the fission, the nature of this coupling is hotly debated. The proposed mechanisms include the 'direct' two-body coulomb coupling ${ }^{24}$ and the presence of an intermediate double triplet state which is degenerate with the singlet exciton $^{25,26}$. Recent studies propose that the 'indirect' coupling, mediated by the mixing of the singlet and triplet excitations with charge-transfer excitons dominates the interaction, leading to reported coupling values in the range $0.02-0.11 \mathrm{eV}\left(200-900 \mathrm{~cm}^{-1)}{ }^{14}, 18,24\right.$ There is some debate over the energies of the charge-transfer states and whether they participate as actual 
intermediates in the SF process, or as virtual states that mediate effective coupling of $S$ and $T T$ via a type of 'super-exchange' ${ }^{15}$ Some recent theoretical work has also begun to evaluate the influence of molecular motion in modulating the coupling between $S$ and $T T .^{18,19}{ }^{27}$ In contrast to the wealth of theoretical studies, there are very few 25,262829 experimental studies that provide an insight into the nature of the $T T$ state, the couplings between the singlet and TT manifolds, or how these couplings are affected by molecular motions. To judge the reliability of theoretical methods and to build up a comprehensive mechanistic picture of SF, new types of experiments accessing both the electronic and vibrational phenomena are needed.

Here we use ultrafast 2D electronic photon echo spectroscopy (2DES) to access both the electronic and coupled vibrational dynamics of SF in a series of pentacene derivatives. The unique transition-pathway selectivity and high time resolution $(<15 \mathrm{fs})$ of this technique allow us to observe a number of previously experimentally inaccessible intermediates in the SF process. Employing a theoretical model, which reproduces the 2DES data, we show that vibrational modes serve to couple the 'bright' $S$ and 'dark' $T T$ excitons, generating a vibronic manifold of mixed singlet/triplet-multiexciton states that mediate the ultrafast SF process.

\section{Results}

Our experimental approach is based on 2DES spectroscopy - a four-wave mixing technique, in which three pulses interact with the system to produce a fourth 'signal' field, interferometrically detected in a phase-matched direction. ${ }^{30}$ The 2DES used here combines sub 15-fs time resolution with a high selectivity in excitation $\left(\omega_{e x}\right)$ and probe $\left(\omega_{p r}\right)$ light frequencies, in contrast to pump-probe spectroscopy that does not allow resolution on the excitation frequency. This allows for selective discrimination of the responses related to different excitation pathways, addressing the effects of electronic and vibrational coherences and, therefore, provides more extensive information about the underlying photophysics. ${ }^{31-39}$

We study pentacene and its derivatives 6,13-di(2'-thienyl)pentacene(DTP) and 6,13bis(triisopropyl-silylethynyl) pentacene(TIPS) as model systems for ultrafast fission, all of which undergo SF on the $\sim 100$-fs timescale. ${ }^{11}$ The ultrafast nature of the process is important as it allows SF to outcompete other single exciton relaxation channels such as radiative decay, excimer formation, intersystem crossing and charge transfer. Figure 1a shows the molecular structure and energy levels of the materials. These molecules, as shown in figure $1 \mathrm{~b}$, share the pentacene aromatic core but have different side groups, which cause them to pack differently in the solid state leading to the different intermolecular couplings. ${ }^{11}$ Material absorption 
spectra, shown in Figure 1c, indicate substantial differences in the energy of singlet states. Based on the absorption data, the lowest singlet state lies at $1.75 \mathrm{eV}$ for TIPS, $1.83 \mathrm{eV}$ for pentacene and $1.86 \mathrm{eV}$ for DTP. The energy of the triplet state is however not expected to differ for these systems, since the triplet is localised on the aromatic core of a single pentacene molecule. ${ }^{40}$ This energy has previously been measured to be $0.86 \mathrm{eV}$ and the energies of the double-triplet $T T$ state for all the systems are therefore expected to lie around $1.72 \mathrm{eV} .{ }^{24}$ Figure 1c also shows the spectrum of the 15-fs laser pulse used in the experiments. The spectrum is broad enough to cover the regions of singlet absorption for all the pentacene derivatives and the spectral region corresponding to the predicted energy of the TT state.

Figure 2 presents three time frames showing the evolution of the real part of the 2DES spectra for a polycrystalline pentacene film (for an evolution of 2D spectra with time see Supplementary Video S1). The sample was tilted by $\sim 50$ degrees with respect to the incident beam to enhance the response of the triplet component, as has been previously discussed. ${ }^{41}$ The 2D spectra are dominated by a large positive peak on the diagonal at $1.83 \mathrm{eV}$, which corresponds to ground-state bleach (GSB) of the main singlet transition $g-S$ and at short times ( $<90 \mathrm{fs}$ ) also includes a contribution from stimulated emission (SE) from the singlet exciton. ${ }^{42}$ At $30 \mathrm{fs}$, a photoinduced absorption (PIA) feature, seen as negative peak at $1.85 \mathrm{eV}$ probe energy, is present. We assign this feature to the excite-state absorption (ESA) of singlet excitons $S-S_{n}$ in agreement with previous pump-probe measurements. ${ }^{41}$ At longer waiting times, this ESA is lost as singlets undergo fission and two new PIA features at $1.70 \mathrm{eV}$ and $1.88 \mathrm{eV}$ probe energy appear. These new peaks are assigned to the transition from the lowest triplet state $T$ to the higher lying $T_{2}$ and $T_{3}$ states respectively. ${ }^{42}$ Importantly, no feature is observed at excitation energy of $1.72 \mathrm{eV}$ where the $T T$ state is predicted to be. The signal is close to zero at all evolution delay times, which indicates that negligible response cannot be a result of interference between singlet and triplet contribution. This confirms that the $T T$ state has negligible (much smaller than $S$ ) transition dipole. The spectra for TIPS and DTP are very similar with the singlet peak shifted in agreement with the absorption spectra (Supplementary Figures S12 and S13).

To confirm the identification of different contributions to the signal and to determine the interconversion rates between different electronic states we de-convolute the $3 \mathrm{D}\left(\omega_{e x}, \omega_{p r}\right.$, $T_{\text {evolution }}$ ) data matrix into $2 \mathrm{D}$ decay-associated spectra. For pentacene, the analysis reveals three components with the characteristic time scales of $14 \mathrm{fs}, 83 \mathrm{fs}$, and $>>1$ ps (Supplementary Figure S14). The fastest timescale corresponds to pulse overlap situation and is assigned to the 
coherent artefact. The spectral configuration of the long-lived component match the spectra of triplet previously observed in pump-probe measurements. ${ }^{42}$ Finally, the spectra and decay time of the 83 fs component reflect the singlet to triplet conversion ( $S$ and $T$ response difference) and are in excellent agreement with previous pump-probe studies, which assign the observed 83 -fs time constant to SF rate. ${ }^{41}$ Kinetics for TIPS and DTP are very similar with SF rates of 100 fs and 130 fs.

Figure $3 \mathrm{a}$ shows the representative kinetics of the real part of the $2 \mathrm{D}$ spectra of pentacene (Figure 2), corresponding to (i) $g-S \mathrm{GSB} / \mathrm{SE}$, (ii) $T-T_{2} \mathrm{ESA}$, and (iii) at $\omega_{e x}=\omega_{p r}=1.72 \mathrm{eV}$ which is the expected position of $g-T T$ GSB. The red curve represents a global fit to the data using the three components described above. The model reproduces the data well, except for the oscillations which can be clearly seen in all three traces. The oscillations are found to be present even at $(1.72 \mathrm{eV}, 1.72 \mathrm{eV})$. At this position the average population is negligible and the signal beats around zero. The frequency of the oscillations does not match an energy difference between electronic states, indicating that these oscillations do not arise from purely electronic coherence. Alternately, the beatings could be caused by shortpulse induced superposition of vibronic states in the electronic ground or excited states manifolds and carry information about the coupled electron-nuclear dynamics.

The beatings seen in Figure 3a are long-lived, lasting over $1500 \mathrm{fs}$, which suggests that they are likely to originate from the vibrational dynamics, ${ }^{32,33,43}$ as electronic dephasing times in molecular systems are typically less than $100 \mathrm{fs}$ at room temperature. ${ }^{44}$ To explore this further, we Fourier transform (FT), along the $T_{\text {evolution }}$ axis, the residual obtained by subtracting the global fit (red curves) from the experimental data (blue curve). The FT spectra obtained for pentacene, TIPS and DTP (Figure 3b, blue curves) show a clear structure with up to ten peaks having a bandwidth of $<20 \mathrm{~cm}^{-1}$. The red curves in Figure $3 \mathrm{~b}$ present steady state resonant Raman measurements for each material. All the peaks in the FT spectra are found to correlate well with the modes observed in the Raman measurements. Additionally, the shape and amplitude of the FT spectra do not change dramatically in time for any of the three materials. Crucially, there are no features in the FT spectra that could correspond to the energy difference between $S$ and $T T, 0.11 \mathrm{eV}=890 \mathrm{~cm}^{-1}$. Hence, there is no evidence for long-lasting electronic coherence between $S$ and $T T$ mediating the singlet fission process. Thus, the comparison between the FT of the oscillatory part of 2DES response and the Raman spectra gives a strong indication of the vibronic origin of the beatings observed. 
The high quality of the experimental data allows us further analyse the detected beatings and achieve additional insight into excited- and ground-state vibronic dynamics. For this, we chose the most pronounces vibrational modes (shown in fig. 3b) and, for each of them, plot a 2D map of the amplitude of the oscillatory component of the response, as a function of excitation and probe frequency $\left(\omega_{e x}\right.$ and $\left.\omega_{p r}\right)$. This yields the $2 \mathrm{D}$ 'beating-maps ${ }^{, 32}$ that are shown in Figure 4 for $265 \mathrm{~cm}^{-1}, 605 \mathrm{~cm}^{-1}, 1170 \mathrm{~cm}^{-1}, 1360 \mathrm{~cm}^{-1}(0.032 \mathrm{eV}, 0.075 \mathrm{eV}, 0.145 \mathrm{eV}$, $0.169 \mathrm{eV}$ ) vibrational modes observed in the FT and resonance Raman spectra of pentacene film (Figure 3b). Similar maps for TIPS and DTP are presented in the Supplementary Figure S15.

For all the 2D beating maps presented in Figure 4 an intense peak is found in the 'diagonal' region of $\omega_{e x}=\omega_{p r}=\omega_{S}=1.83 \mathrm{eV}$, which corresponds to the lowest singlet exciton level. In addition, multiple off-diagonal peaks are observed at $\omega_{e x}=1.83 \mathrm{eV}$. As shown by the arrows in Figure 4, these peaks are displaced from the diagonal peak along the probe axis roughly by the energy corresponding to the vibrational mode considered in the beating map. ${ }^{32}$, ${ }^{33}$ For example, in the $605 \mathrm{~cm}^{-1}$ (Figure $4 \mathrm{~b}$ ) beating map peaks can be seen at probe frequency $\omega_{p r}=\omega_{S}-605 \mathrm{~cm}^{-1}$ while the $1170 \mathrm{~cm}^{-1}$ beating map shows a peak at $\omega_{p r}=\omega_{S}-1170 \mathrm{~cm}^{-1}$ (figure 4c). These and additional peaks that occur below and to the right of the diagonal (dashed line in figures) are caused by the coupling of vibrational modes to an electronic transition. The exact shape of the beating pattern is complex and different for the rephasing and non-rephasing parts of 2DES response. However, our observation of beating peaks for $\omega_{\mathrm{ex}}>=\omega_{S}(1.83 \mathrm{eV})$ can be described within the theoretical framework considering vibrations coupled to a single electronic state. ${ }^{32,33,43}$

The above described peak pattern for $\omega_{e x}>=\omega_{S}(1.83 \mathrm{eV})$ is associated with the GSB signal and, as such, predominantly reflects the coherent vibrational dynamics in the groundstate. ${ }^{33}$ However, the beating maps in figure 4 clearly contain more peaks, especially for the high-frequency $1170 \mathrm{~cm}^{-1}$ and $1360 \mathrm{~cm}^{-1}$ oscillatory modes. Most of these additional peaks are located at the excitation frequency $\omega_{e x} \sim 1.72 \mathrm{eV}$ which is below the singlet transition and therefore cannot be described by GSB signals and ground-state coherences from the singlet vibrational manifold. However, this energy coincides with the expected position of the TT state, suggesting that the additional peaks could be associated with transitions from the ground to multiexciton state. Such a transition would have a transition dipole much smaller than that of the singlet excitation, making it indistinguishable in the linear absorption spectrum, where it 
would be obscured in the tail of the $S$ absorption feature. However, as the intensities of the peaks in beating maps are determined by four different interactions with the field, ${ }^{43}$ even if one of the transition dipoles is very low (i.e. a 'dark' transition), the product of interactions and the intensity of the beating peak can be significant. In essence, the vibrational dynamics presented in beating maps may reveal transitions normally hidden in linear absorption or smeared out in the measurements addressing population dynamics. ${ }^{32,33,35}$

To unravel the rich data provided by the beating maps, on the coupling between electronic states and vibrational modes, we construct a model that explains, qualitatively and quantitatively, all the features observed in the beating maps, and reproduces the experimental 2DES dataset. Here we will qualitatively describe the model while a detailed quantitative picture is presented in part S.1 of The Supplementary Information . First of all, the involvement of the $T T$ state in the formation of beating pattern implies that coherent superpositions of $T T$ and some other states, displaced by certain vibrational frequencies, are being excited. These other states can be assigned to the vibronic multiexciton manifold (TT') (Figure 1). Importantly, some states within this manifold have energy close to that of the singlet; for pentacene these states are $T T^{\prime}{ }_{1170}$ and $T T^{\prime}{ }_{1360}$, which lie at $T T+1170 \mathrm{~cm}^{-1}$ and $T T+1360 \mathrm{~cm}^{1}$ respectively (Figure 1a). This allows for partial mixing of the singlet and multiexcitons $T T^{\prime}$ ' even by means of modest electronic coupling $\left(248 \mathrm{~cm}^{-1}\right)$. Such mixing may not substantially alter $T T^{\prime}{ }_{1170}$ and $T T^{\prime}{ }_{1360}$ energies, but it may make those states 'bright' and observable in the beating spectra.

Figure 5a,b explains the different contributions to the $1170 \mathrm{~cm}^{-1}$ and $1360 \mathrm{~cm}^{-1}$ rephasing/non-rephasing beating maps using the assumptions discussed above. Optical transitions occur between four electronic states: the ground state g, the lowest singlet $S$, multiexciton $T T$, and $T T_{3}$ - an excited multiexciton state formed via the $T-T_{3}$ transition for one of the triplets, which is known to lie at $2.38 \mathrm{eV}$ for pentacene. We also consider the vibrationally hot ground $g$ ' and mulitexciton $T T^{\prime}$ states. Figure $5 \mathrm{~b}$ depicts the four-wavemixing pathways where a superposition of states is created after the first two interactions and mark the corresponding peak positions in the beating maps. Finally, we consider the initial thermal population of vibrationaly excited ground states $g$ ' negligible.

Within the above framework, we can describe all the peaks in Figure 5a. Peaks 1-2 are due to the ground-state vibrational coherence and GSB of $S$ state. Peaks 3-4 correspond to creating a coherent superposition in the excited state, between $T T$ and $T T^{\prime}$ (mixed with $S$ ), which is observed through the transition to a higher-lying $T T_{3}$ state. As seen from the diagrams, at least three out of four interactions involve bright states; this makes the total amplitude of the 
beating peaks significant. Thus, the proposed qualitative model accurately explains the peaks in the beating maps, providing strong evidence that our observations reveal a vibronic manifold of mixed $S$-multiexcitonic states.

This interpretation is supported by the appearance of additional peaks 3,4 only for highfrequency vibrations. As shown in Figure 1a the $T T, T T^{\prime}{ }_{265}$ or $T T^{\prime}{ }_{605}$ states are energetically much further from $S$ than $T T^{\prime}{ }_{1170}$ and $T T^{\prime}{ }_{1360}$, which prohibit them from 'borrowing' the oscillator strength and keeps them dark. This leads to the disappearance of peaks 3-4 from $265 \mathrm{~cm}^{-1}$ and $605 \mathrm{~cm}^{-1}$ beating patterns as observed in figure 4 . Consistent with the above interpretation, figure 5c shows that in the case of TIPS (but not DTP) the 3-4 beating features appear for the lower $265 \mathrm{~cm}^{-1}$ vibrational mode, as the smaller gap between $S$ and TT brings the $T T^{\prime}{ }_{265}$ mode almost in resonance with the TIPS singlet exciton (fig.1a).

To confirm the assignment of the observed coherences to the singlet-multiexciton mixed excited states and support the proposed interpretation, we have undertaken a set of complementary experiments and analysis, developed to identify the nature of coherent states. While detailed results and discussions can be found in Supplementary Information parts S.2.7S.2.9, here we summarise the main findings. A phase analysis ${ }^{45}$ of the beatings confirms the excited-state nature of peaks 3 and 4 and the ground-state origin of peaks 1 and 2. Polarisationselective measurements ${ }^{46}$ reveal that the beating features 3 and 4 for high-frequency vibrations are associated with mixed electronic states, e.g. singlet-multiexciton mixtures. Finally, complimentary 2D measurements in a different spectral range, 2-2.4 eV, support the assignment of ESA features and confirm the existence of long-lived excited state coherence, coming from the mixed vibronic states.

\section{Discussion}

To quantitatively extend the analysis, we construct a vibronic model of the observed SF dynamics. This model can quantitatively reproduce the 2D photon-echo data, including the beating map patterns. Importantly, the same set of parameters capture the vibronic level structure, the spectroscopic observables and the ultrafast dynamics of the SF process. The model consists of three Raman active modes $\left(265 \mathrm{~cm}^{-1}, 1170 \mathrm{~cm}^{-1}\right.$, and $1360 \mathrm{~cm}^{-1}$ with HuangRhys factors $0.7,0.6$ and 0.75 respectively) and two coupled electronic states. One of the electronic states is assumed to be optically bright and represents the $S$ singlet electronic excitation coupled to the Raman modes. The second electronic state is chosen as optically dark and represents the multiexciton state. The electronic coupling between singlet and multiexciton 
manifolds employed in the model $\left(248 \mathrm{~cm}^{-1}=0.031 \mathrm{eV}\right)$ is small compared to the energy difference between the $S$ and $T T$ states $\left(\sim 900 \mathrm{~cm}^{-1}=0.11 \mathrm{eV}\right)$. To adequately model the spectroscopic response, the electronic ground state as well as a higher-lying electronic state (ESA occurs to this state) with their vibrational manifolds are explicitly considered as well, although they do not have any impact on the excited-state dynamics relevant for the fission process. We also introduce a weak linear coupling to a harmonic bath, as described within the multilevel Redfield approach. ${ }^{47}$ Full details of the model can be found in the Supplementary Information parts S.1.1-6.

Using the above parameters we modelled the structure of the vibronic manifold, 2DES beating maps, and the population kinetics for the excited states during SF in pentacene. Partial mixing of $S$ and $T T$ vibronic manifolds leads to the formation of a dense manifold of 16 new eigenstates (figure 6a). The lowest four possess mostly (95\%) multiexciton contribution, the fifth lowest state is dominantly singlet, and higher-lying states have a more mixed character. The dipole moments of these states vary significantly, however even the lowest state (close to diabatic TT) is not completely dark. The calculated oscillator strength agree well with the absorption spectrum of pentacene (figure $6 \mathrm{~b}$ ), indicating that the 'dominantly-multiexcitonic' absorption feature is likely to be present, but hidden under the broad and intense 'dominantlysinglet' features. The modelled beating maps are also in a good agreement with the experimental data (compare figures $6 \mathrm{c}$ and $5 \mathrm{a}$ ), showing that the model accurately captures the underlying photophysical processes. The dynamics produced by the model (fig. $6 \mathrm{~d}$ ) show that the total population of optically bright states decays on an 83-fs timescale, consistent with the singlet-triplet conversion rate reported in previous pump-probe studies. ${ }^{41}$

Interestingly, due to the mixed character of the initially photoexcited states, the total multiexcitonic (dark) population $12 \mathrm{fs}$ (pulse duration) after excitation is significant, which is in good agreement with the near instantaneous rise of a triplet feature observed by Zhu and co-workers using time-resolved photoemission measurements. ${ }^{25,}{ }^{48}$ However, as described above, we attribute this signal to the mixed electronic characters of the vibronic eigenstates, rather than to strong electronic coupling between the singlet and a single degenerate multiexciton state as proposed by Zhu et al. Based on our data, we propose that the cooling process observed by Zhu et al. on a $200-\mathrm{fs}$ timescale corresponds to vibrational cooling of eigenstates of predominantly $T T$ character (Supplementary Informaiton section S.1.3).

The crucial role of the vibronic-manifold of states for fast and efficient SF reveals the general importance of nuclear degrees of freedom for the optoelectronic properties of organic 
materials. In contrast to the classical inorganic semiconductors, molecular systems suffer from weak intermolecular interactions and high disorder, which prevents the formation of delocalised all-electronic band states. However, coupling of the electronic system to multiple intramolecular vibrational modes creates vibronic manifolds with a high density of states. These dense manifolds can facilitate efficient coupling between different molecules (or intramolecular states) even when only weak interactions are present. Indeed, coherent vibronic phenomena has recently been proposed to be crucial to electronic processes in organic photovoltaics $^{49}$ and natural photosynthetic systems. ${ }^{50}$

In conclusion, using 2DES, we observed a manifold of mixed singlet/multiexcitonic states mediating ultrafast SF in pentacene and its derivatives. Our observations and modelling indicate that overlap and mixing of the vibronic manifolds of singlet exciton and multiexcitonic state play a key role in ultrafast dynamics of SF. In particular, the presence of these mixed states means that strong electronic couplings, exceeding the energy difference between the singlet and the multiexcitonic state, are not required to allow for sub $100 \mathrm{fs}$ conversion between singlet and triplet states. Our data not only provide firm experimental evidence of the importance of vibronic coupling and mixed intermediate states in the fission process, but may also serve as the basis for future theoretical models of the photophysics of a broad class of organic chromophores that could allow such mixed states.

\section{Methods:}

\section{D spectroscopy:}

A solid state KGW amplified laser system (Pharos, Light Conversion) pumped a homemade NOPA producing 14-fs pulses centered at $14500 \mathrm{~cm}^{-1}\left(\mathrm{FWHM} 1800 \mathrm{~cm}^{-1}\right)$. Pharos operated at $200 \mathrm{kHz}$ and an internal pulse picker was used to reduce the repetition rate to $20 \mathrm{kHz}$. Each pulse was split into four by a beamsplitter and a transmissive diffractive grating. The population delay between pulses 2 and 3 was set by a conventional optical delay line and the coherence delay between pulses 1 and 2 was set by inserting variable amount of fused silica in their optical paths. One beam, serving as the local oscillator (LO), was further attenuated by an OD 3 neutral density filter, and all four pulses were focused in boxcar geometry on a single spot at the sample. Photon echo type signal is emitted into the phase matching direction coinciding with the direction of LO, and spectral interference between them is detected by the combination of the spectrometer and CCD camera. Two of the excitation pulses were modulated by 
optomechanical choppers operating at different frequencies. Lock-in detection on the sum and difference frequency was used to discriminate the signal against scattered light. During the experiments the time delay between the first two pulses (coherence time) was scanned from 200 fs to 200 fs with the 2 fs steps. The absorption spectrum of the sample was controlled during the measurement by monitoring the spectrum of LO passing through the sample. No changes were observed during the experiments, therefore indicating that the sample was stable during the measurements.

\section{Raman spectroscopy}

Stokes-shifted Raman spectra of pentacene and derivatives films were recorded using a Horiba T64000 Raman system Helium-neon laser line at $632.8 \mathrm{~nm}$ was used for excitation, which was focussed on to the TIPS-pentacene film with a Leica $50 \mathrm{x}, 0.55$ numerical aperture, long working-distance microscope objective. The laser power at the sample was approximately 130 $\mu \mathrm{W}$. The sample was placed in a Linkam chamber through which a small flow of nitrogen gas was maintained.

\section{Sample preparation}

6,13-bis(triisopropylsiylethynyl)pentacene [CAS 373596-08-8] >99\%, (TIPS-pentacene) was purchased from Sigma Aldrich and used as received. The material was dissolved in toluene (5 $\mathrm{wt} \%$ ) and spin-cast onto quartz substrates at $1500 \mathrm{rpm}$, in a nitrogen glovebox. Polycrystalline pentacene and 6,13-di(2-thienyl)pentacene (DTP) films $150 \mathrm{~nm}$ thick were deposited via vacuum sublimation at a rate of $0.4 \AA / \mathrm{s}$ in a vacuum of $5 \times 10^{-6}$ mbar or better. All samples were encapsulated in a nitrogen glove box before spectroscopic measurements.

\section{Excited-state dynamics and 2D spectra calculations:}

The dynamics simulations have been performed in the eigenstate basis of the model Hamiltonian using multi-level Redfield approach, the interaction with the excitation pulse of 13 fs duration (Gaussian envelope) has been explicitly included (linear dipole approximation). Bilinear interaction with an Ohmic harmonic bath has been assumed. The eigenstates have been used to calculate the response functions corresponding to the 2DES signal in the stick-spectrum limit. To obtain finite line widths, Lorentzian lineshapes have been employed, with dephasing rates estimated from Redfield dynamics. All pathways involving transitions where the energy difference between the states falls outside of the laser spectrum were excluded. A detailed discussion of theoretical methods and parameters is given in Supplementary Information S1. 


\section{Acknowledgements:}

We thank Thomas Jansen and Maxim Pschenichnikov for useful discussions, David Palecek for the help with $\sim 580 \mathrm{~nm}$ 2D experiments and Maxim Tabachnyk for the help with preparing samples. This work was supported by Laserlab-Europe (Project:LLC001945). A.A.B. is currently a Royal Society University Research Fellow, he also acknowledges a Veni grant from the Netherlands Organization for Scientific Research (NWO). A.R., S.E.M. and A.W.C. thank the Winton Programme for the Physics of Sustainability for support.

\section{Author contributions:}

A.A.B. and A.R. conceived the study. M.W.B.W and A.R. produced and charecterised the samples. A.A.B., D.Z. and A.R. planned and performed the 2D experiments. T.K. performed cw-Raman experiments. A.A.B., S.E.M., A.W.C., D.E., and A.R. analysed the data. S.E.M., A.W.C., and D.E. developed the model and performed theoretical calculations. A.A.B., S.E.M., A.W.C., D.E., and A.R. wrote the paper with input from all the authors.

\section{TOC figure}
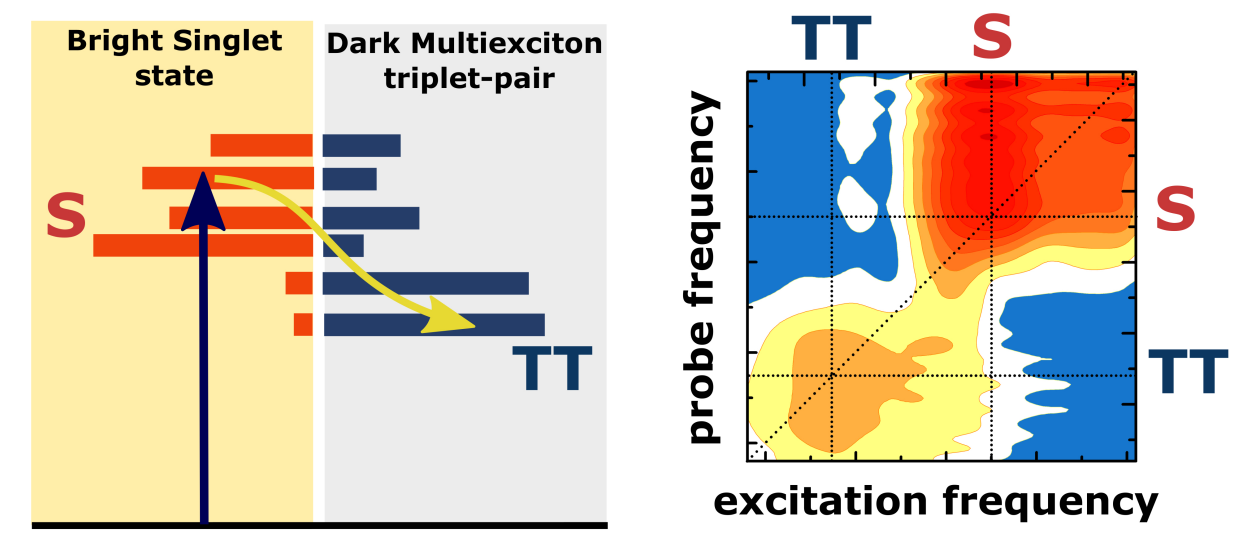

\section{TOC summary}

Singlet fission, a spin-allowed conversion of a spin-singlet state into a pair of spin-triplet excitons, may be useful for the development of next generation photovoltaics. Ultrafast coherence measurements show that vibrational motions play a critical role in fission as they facilitate the mixing of triplet-pair states with singlet excitons. 

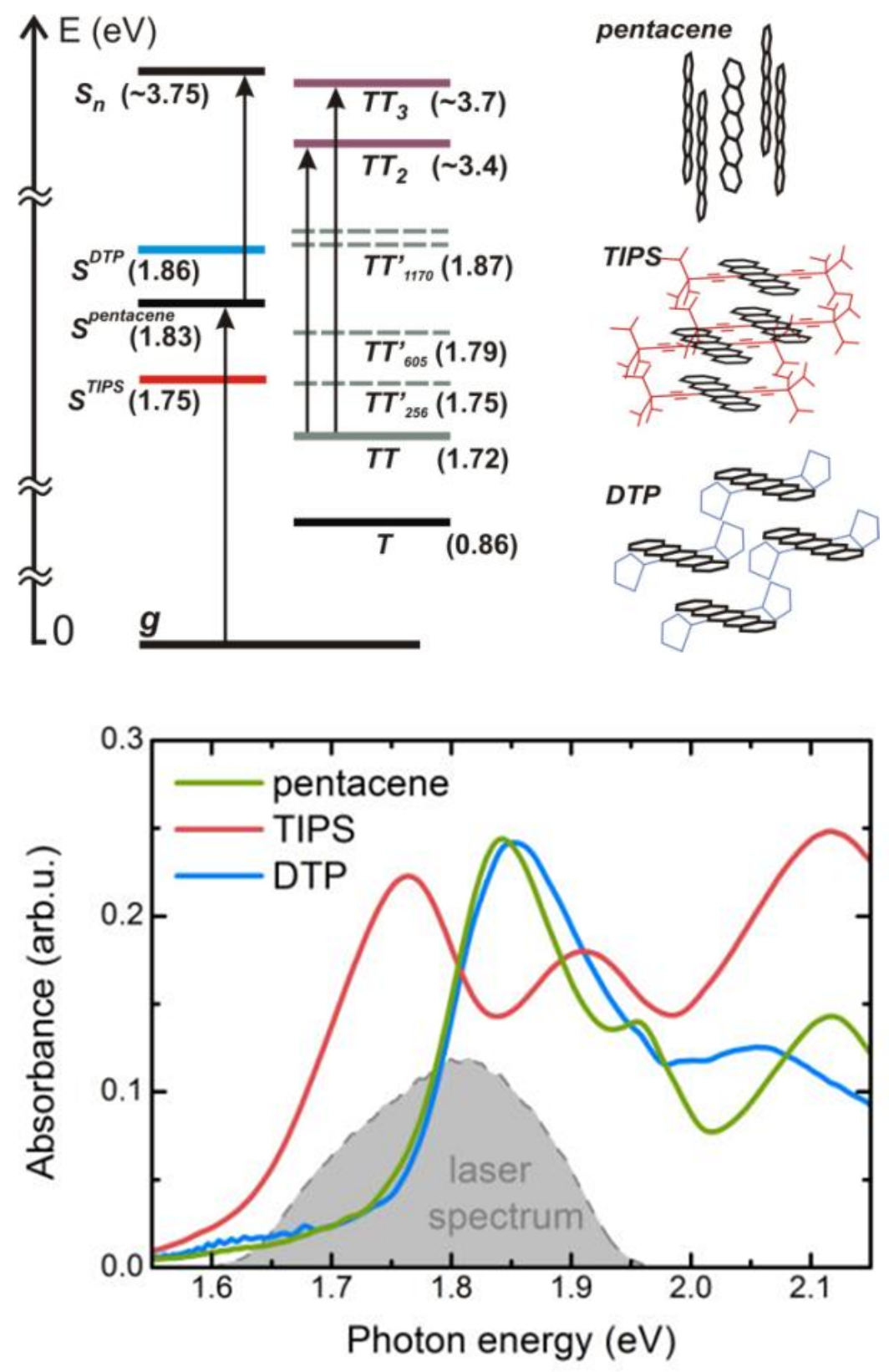

Figure 1. The molecular crystals under study. a. The free-energy diagram depicting electronic and vibronic (marked with ') states addressed in the 2DES experiments. S, T and $g$ correspond to singlet, triplet and ground states respectively. The parenthesis show approximate energies of the states in $\mathrm{eV}$. b. The molecular-crystal structure of the systems under study. $\boldsymbol{c}$. The absorption spectra of the films under study. The grey contour presents the laser spectrum used in 2DES experiments. 

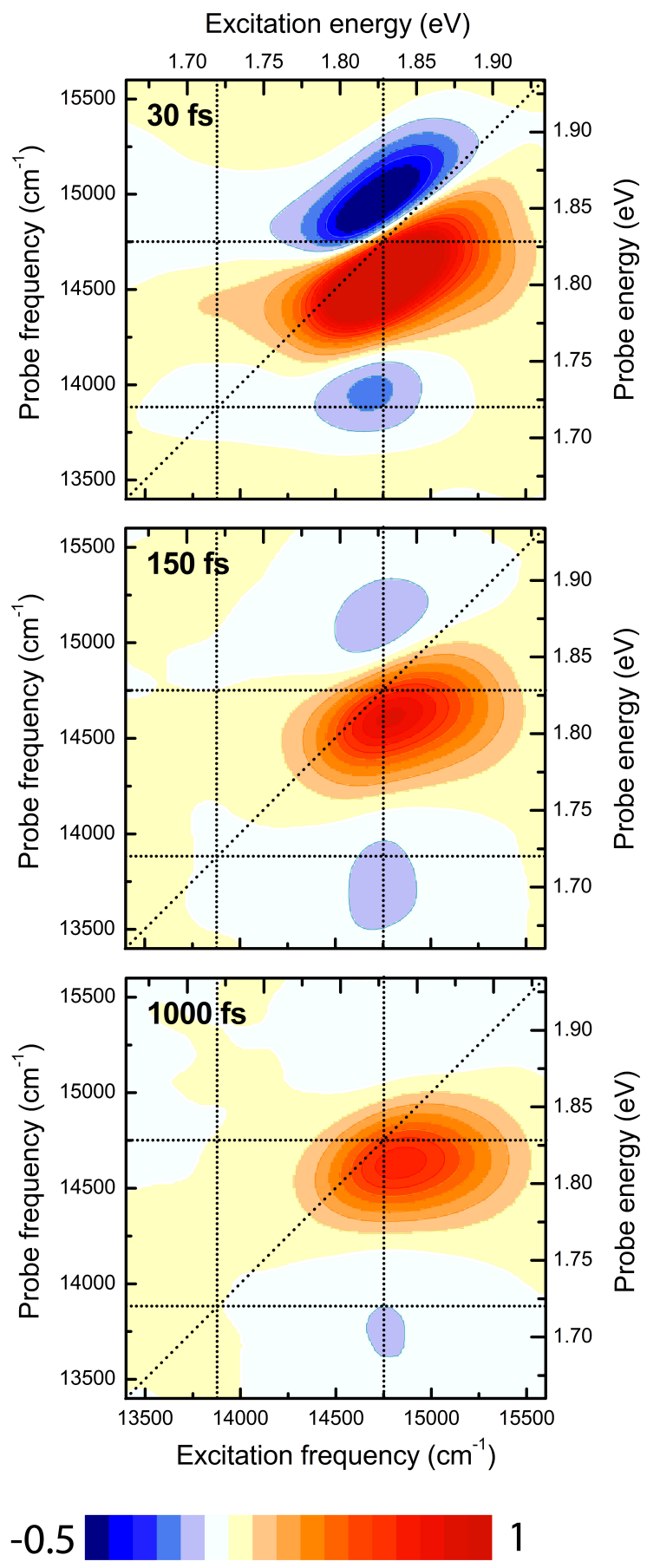

Figure 2. The real part of 2DES spectra for pentacene molecular crystal at different evolution times. Red peaks represent photoinduced increase in transmission due to groundstate bleach and stimulated emission. Blue peaks correspond to photoinduced absorption. While at short evolution time spectra contain mostly singlet features, at longer times the response becomes more triplet-like. Horizontal and vertical dotted lines mark the expected of singlet and multiexciton energies according to the state diagram in figure 1 a. 

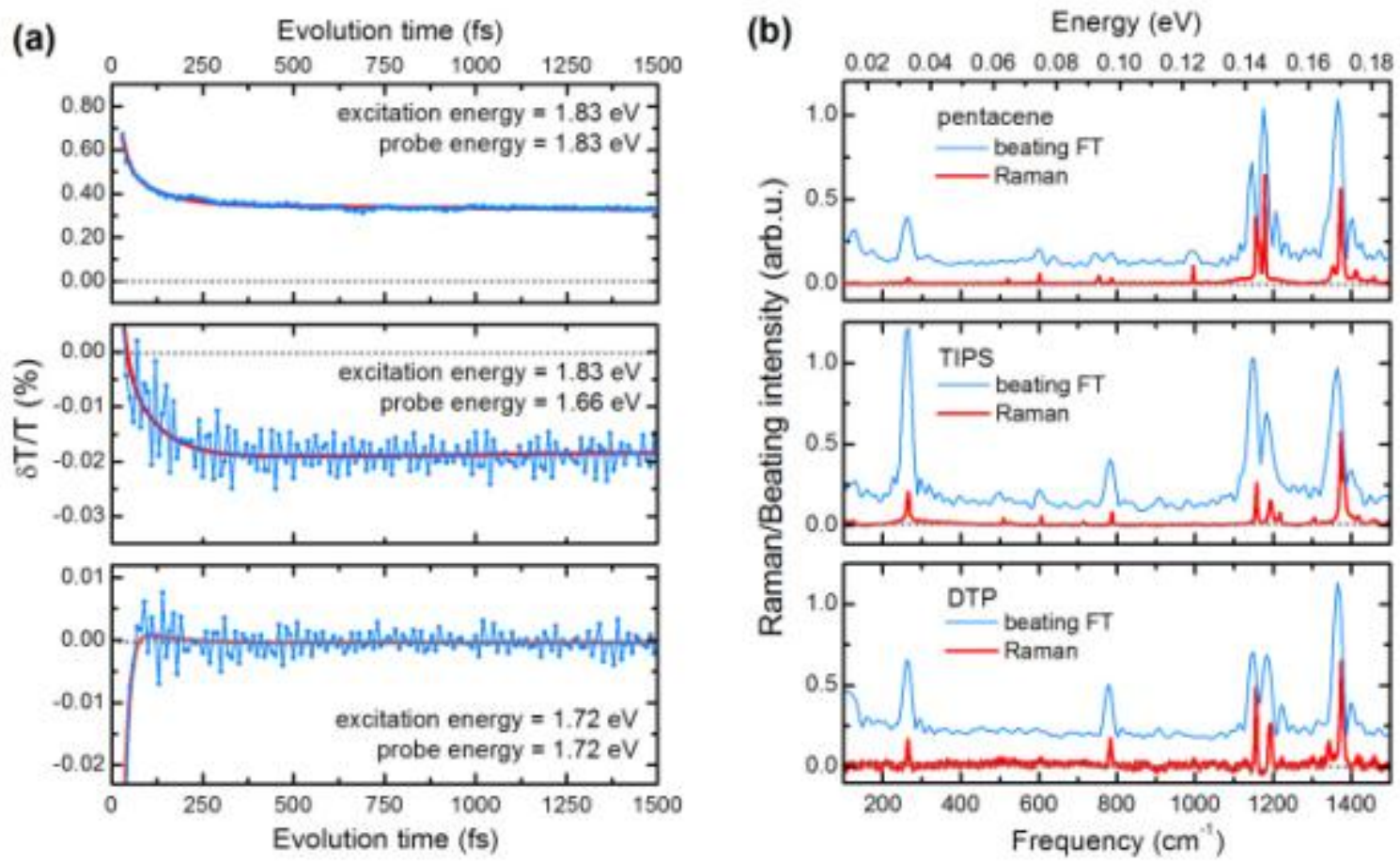

Figure 3. The oscillatory components in the 2DES data. a. Blue curves show evolution-time transients corresponding to the different locations in the 2D spectrum of pentacene. Red curve is the description of the population dynamics by the 3-component decay-associated spectra. $\boldsymbol{b}$. Spectra of evolution-time oscillations observed in 2DES data integrated over complete $2 D$ spectra compared to the resonance Raman spectra of the films of pentacene and its derivatives. 


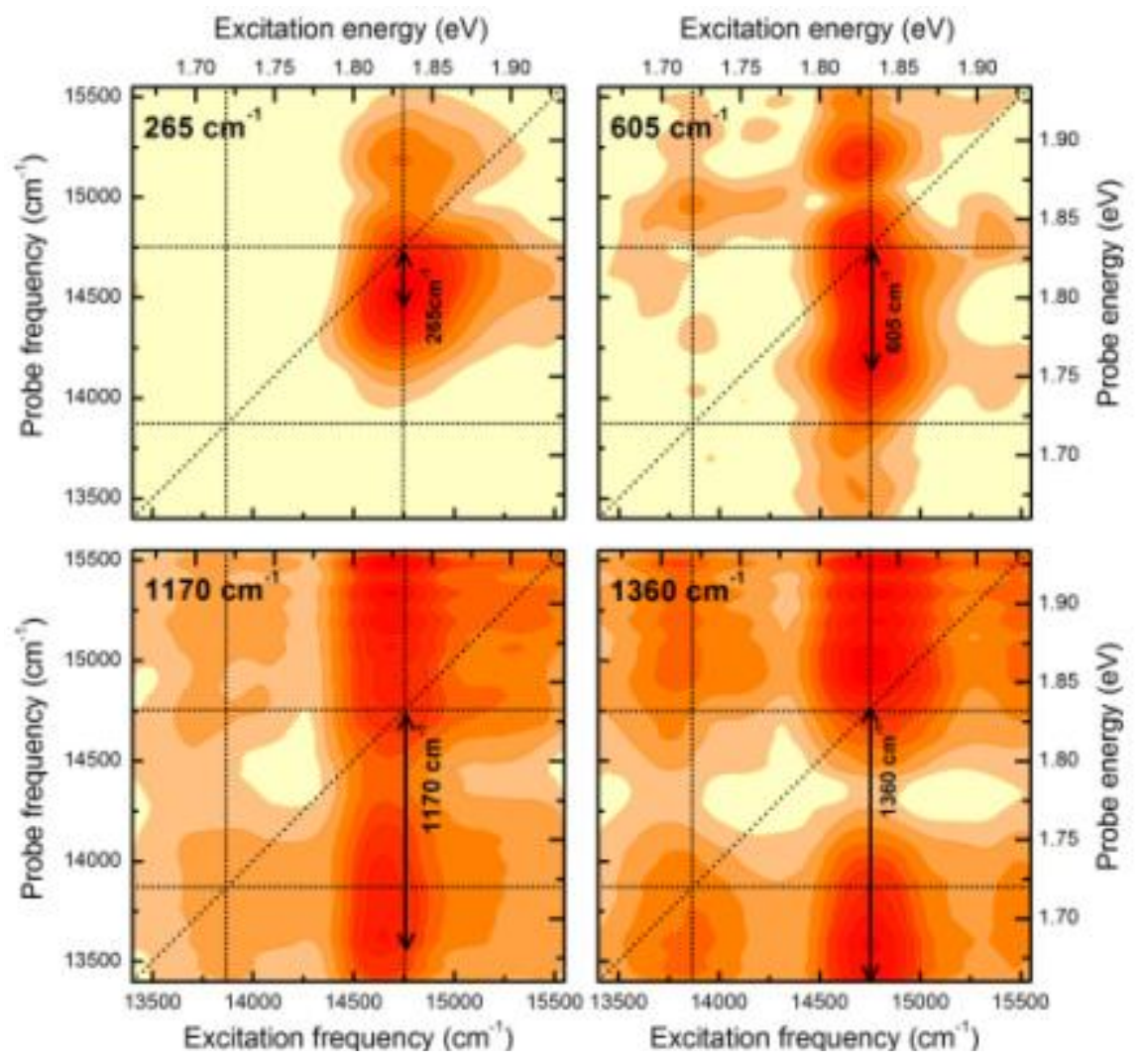

Figure 4. The 2D maps of beatings in 2DES spectra corresponding to the strongest oscillatory features observed in figure $3 b$. The length of the arrow in every panel corresponds to the energy of the corresponding vibrational mode. For low-frequency modes the beating signals are localised around the S state, while for higher-frequency modes coherences are also observed at $\omega_{\text {ex }} \sim 1.72 \mathrm{eV}$ corresponding to the energy of TT state. 
(a)
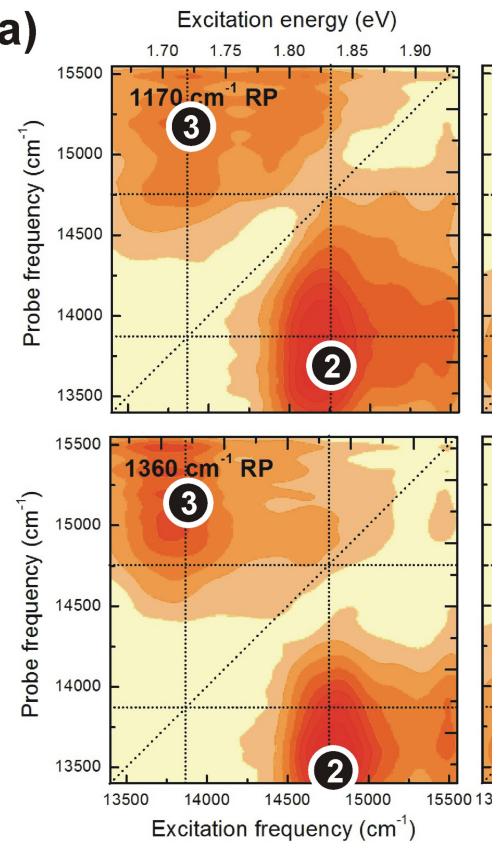

(c)

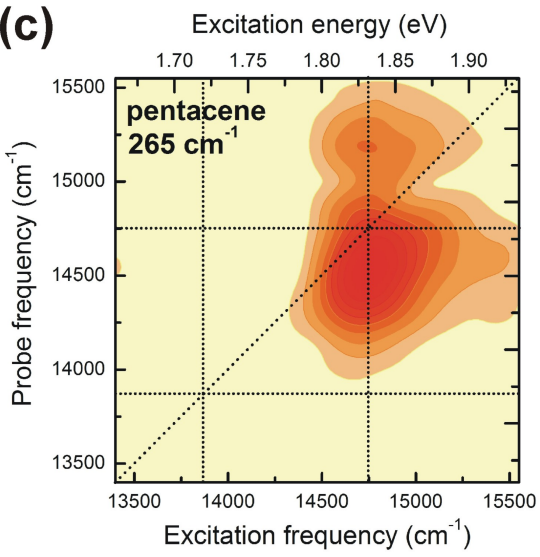

Excitation energy (eV)
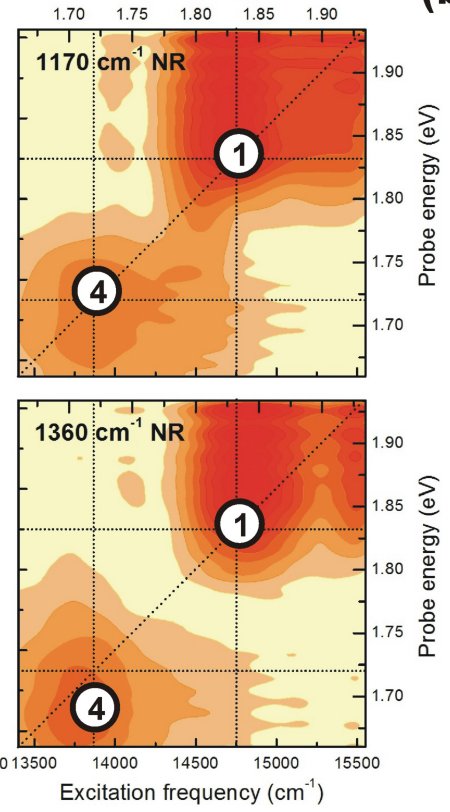

Excitation frequency $\left(\mathrm{cm}^{-1}\right)$

(b)
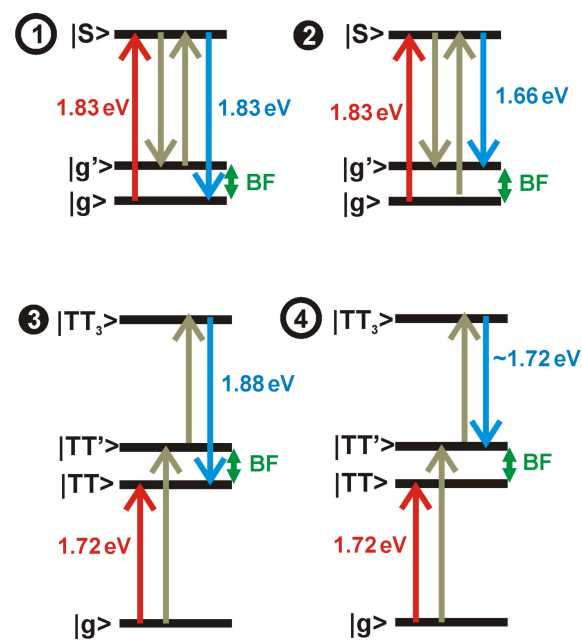

$B F=\begin{gathered}\text { beating } \\ \text { frequency }\end{gathered}$
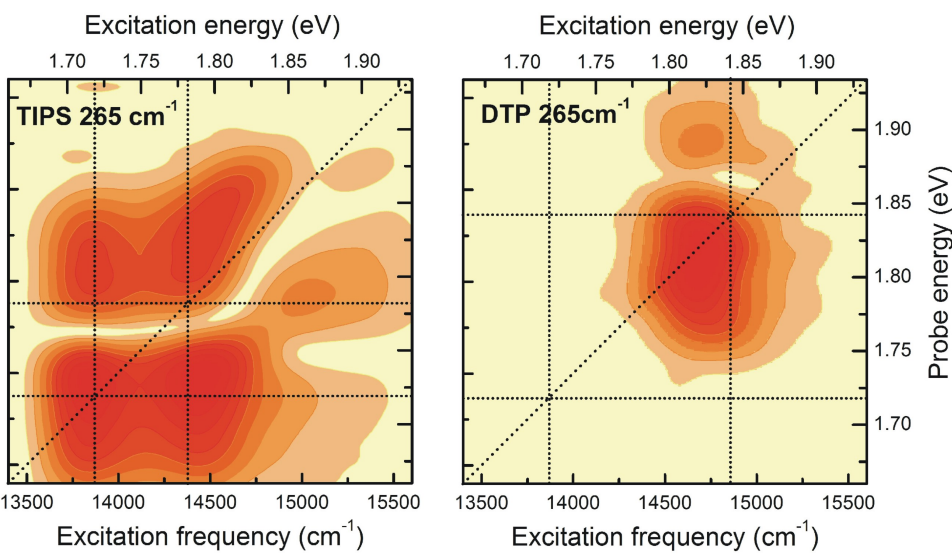

Figure 5. In depth analysis of $2 D E S$ results. (a) The rephasing $(R P)$ and non-rephasing (NR) part of $2 D$ beating maps corresponding to observed high frequency vibrational modes of pentacene and (b) diagrams present the relevant system-field interactions. The numbers mark the corresponding positions of the beating peaks. Rephasing features are marked with black and non-rephasing with white circles. (c) The 2D maps of beating maps corresponding to the $265 \mathrm{~cm}^{-1}$ oscillatory features observed in pentacene and its DTP and TIPS derivatives. 
(a)

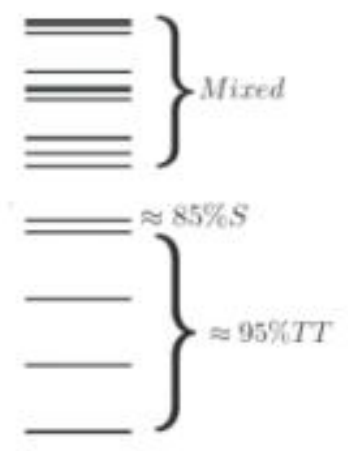

(c)

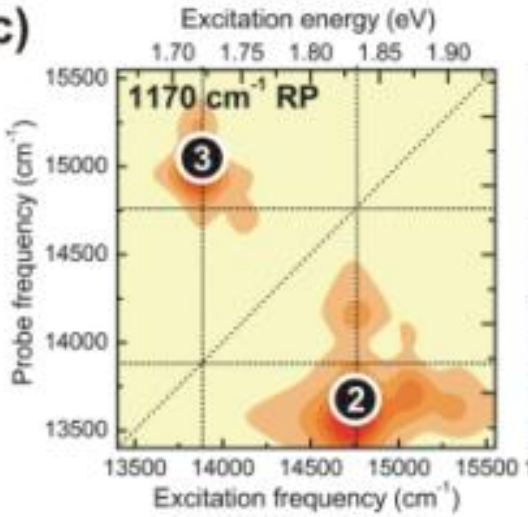

Excitation energy (eV)

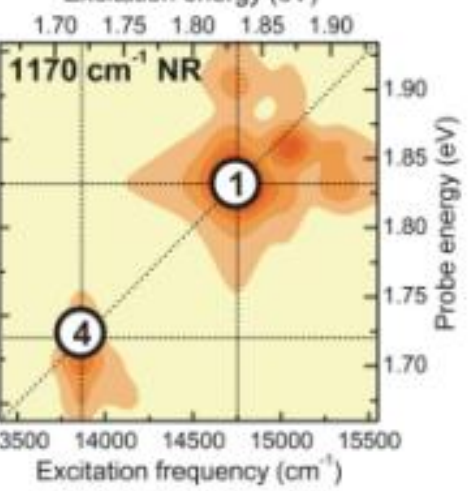

(b)

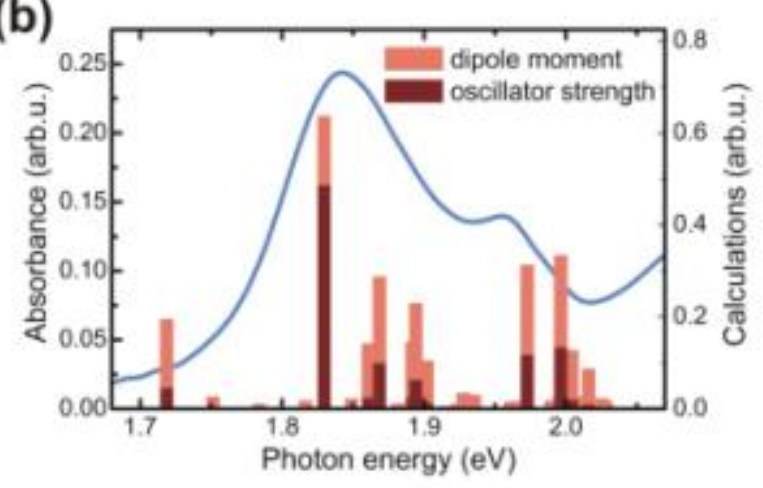

(d)

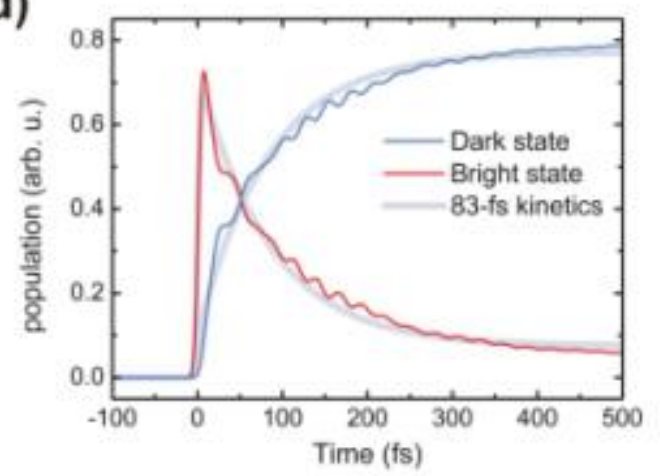

Figure 6. The results of theoretical calculation based on the vibronic state model. (a) The diagram of eigenstates formed as a result of mixing between the singlet and multiexciton manifold. (b) The calculated dipole moments and oscillator strengths for the eigenstates; the blue curve shows an experimental absorption spectrum for comparison. (c) Calculated rephasing/non-rephasing beating maps for pentacene at $1170 \mathrm{~cm}^{-1}$. The same beating features as in the figure 5a,b are shown with black and white circles. (d) The population kinetics for dark and bright states predicted by the model. The grey curves indicated the 83-fs dynamics deduced from the decay-associated spectra analysis (fig. 3a). 


\section{References:}

1. Smith, M.B. \& Michl, J. Singlet Fission. Chemical Reviews110, 6891-6936 (2010).

2. Wilson, M.W.B. et al. Ultrafast Dynamics of Exciton Fission in Polycrystalline Pentacene. Journal of the American Chemical Society133, 11830-11833 (2011).

3. Hanna, M.C. \& Nozik, A.J. Solar conversion efficiency of photovoltaic and photoelectrolysis cells with carrier multiplication absorbers. Journal of Applied Physics100, - (2006).

4. Ehrler, B., Wilson, M.W., Rao, A., Friend, R.H. \& Greenham, N. Singlet Exciton FissionSensitized Infrared Quantum Dot Solar Cells. Nano Letters (2012).

5. Congreve, D.N. et al. External Quantum Efficiency Above $100 \%$ in a Singlet-Exciton-FissionBased Organic Photovoltaic Cell. Science340, 334-337 (2013).

6. Burdett, J.J. \& Bardeen, C.J. The Dynamics of Singlet Fission in Crystalline Tetracene and Covalent Analogs. Accounts Chem. Res.46, 1312-1320 (2013).

7. Dillon, R.J., Piland, G.B. \& Bardeen, C.J. Different Rates of Singlet Fission in Monoclinic versus Orthorhombic Crystal Forms of Diphenylhexatriene. Journal of the American Chemical Society135, 17278-17281 (2013).

8. Mastron, J.N., Roberts, S.T., McAnally, R.E., Thompson, M.E. \& Bradforth, S.E. Aqueous colloidal acene nanoparticles: A new platform for studying singlet fission. Journal of Physical Chemistry B117, 15519-15526 (2013).

9. Lee, J. et al. Singlet Exciton Fission in a Hexacene Derivative. Advanced Materials25, 14451448 (2013).

10. Musser, A.J. et al. Activated Singlet Exciton Fission in a Semiconducting Polymer. Journal of the American Chemical Society135, 12747-12754 (2013).

11. Yost, S.R. et al. A transferable model for singlet-fission kinetics. Nat Chem6, 492-497 (2014).

12. Herz, J. et al. Acceleration of singlet fission in an aza-derivative of TIPS-pentacene. Journal of Physical Chemistry Letters5, 2425-2430 (2014).

13. Busby, E. et al. Multiphonon Relaxation Slows Singlet Fission in Crystalline Hexacene. Journal of the American Chemical Society136, 10654-10660 (2014).

14. Beljonne, D., Yamagata, H., Brédas, J.L., Spano, F.C. \& Olivier, Y. Charge-Transfer Excitations Steer the Davydov Splitting and Mediate Singlet Exciton Fission in Pentacene. Physical Review Letters110, 226402 (2013).

15. Johnson, J.C., Nozik, A.J. \& Michl, J. The Role of Chromophore Coupling in Singlet Fission. Accounts Chem. Res.46, 1290-1299 (2013).

16. Zimmerman, P.M., Musgrave, C.B. \& Head-Gordon, M. A Correlated Electron View of Singlet Fission. Accounts Chem. Res.46, 1339-1347 (2013).

17. Wang, L., Olivier, Y., Prezhdo, O.V. \& Beljonne, D. Maximizing Singlet Fission by Intermolecular Packing. The Journal of Physical Chemistry Letters5, 3345-3353 (2014).

18. Berkelbach, T.C., Hybertsen, M.S. \& Reichman, D.R. Microscopic theory of singlet exciton fission. III. Crystalline pentacene. The Journal of Chemical Physics141, - (2014).

19. Zimmerman, P.M., Zhang, Z. \& Musgrave, C.B. Singlet fission in pentacene through multiexciton quantum states. Nat Chem2, 648-652 (2010).

20. Zeng, T., Ananth, N. \& Hoffmann, R. Seeking Small Molecules for Singlet Fission: A Heteroatom Substitution Strategy. Journal of the American Chemical Society136, 12638-12647 (2014).

21. Coto, P.B., Sharifzadeh, S., Neaton, J.B. \& Thoss, M. Low-Lying Electronic Excited States of Pentacene Oligomers: A Comparative Electronic Structure Study in the Context of Singlet Fission. Journal of Chemical Theory and Computation11, 147-156 (2014).

22. Parker, S.M., Seideman, T., Ratner, M.A. \& Shiozaki, T. Model Hamiltonian Analysis of Singlet Fission from First Principles. The Journal of Physical Chemistry C118, 12700-12705 (2014).

23. Merrifield, R.E., Avakian, P. \& Groff, R.P. Fission of singlet excitons into pairs of triplet excitons in tetracene crystals. Chemical Physics Letters3, 386-388 (1969).

24. Smith, M.B. \& Michl, J. Recent Advances in Singlet Fission. Annual Review of Physical Chemistry64, 361-386 (2013). 
25. Chan, W.-L. et al. Observing the Multiexciton State in Singlet Fission and Ensuing Ultrafast Multielectron Transfer. Science334, 1541-1545 (2011).

26. Chan, W.-L., Ligges, M. \& Zhu, X.Y. The energy barrier in singlet fission can be overcome through coherent coupling and entropic gain. Nat Chem4, 840-845 (2012).

27. Alguire, E.C., Subotnik, J.E. \& Damrauer, N.H. Exploring Non-Condon Effects in a Covalent Tetracene Dimer: How Important Are Vibrations in Determining the Electronic Coupling for Singlet Fission? The Journal of Physical Chemistry A119, 299-311 (2015).

28. Renaud, N. \& Grozema, F.C. Intermolecular Vibrational Modes Speed Up Singlet Fission in Perylenediimide Crystals. The Journal of Physical Chemistry Letters6, 360-365 (2014).

29. Musser, A.J. et al. Evidence for conical intersection dynamics mediating ultrafast singlet exciton fission. Nat Phys11, 352-357 (2015).

30. Jonas, D.M. Two-dimensional femtosecond spectroscopy. Ann.Rev.Phys.Chem.54, 425-463 (2003).

31. Egorova, D. Detection of electronic and vibrational coherences in molecular systems by 2D electronic photon echo spectroscopy. Chem. Phys.347, 166-176 (2008).

32. Butkus, V., Zigmantas, D., Valkunas, L. \& Abramavicius, D. Vibrational vs. electronic coherences in 2D spectrum of molecular systems. Chemical Physics Letters545, 40-43 (2012).

33. Egorova, D. Self-Analysis of Coherent Oscillations in Time-Resolved Optical Signals. The Journal of Physical Chemistry A118, 10259-10267 (2014).

34. Brixner, T., Stenger, J., Vaswani, H.M., Blankenship, R.E. \& Fleming, G.R. Two-dimensional spectroscopy of electronic couplings in photosynthesis. Nature343, 625-628 (2005).

35. Ostroumov, E.E., Mulvaney, R.M., Cogdell, R.J. \& Scholes, G.D. Broadband 2D Electronic Spectroscopy Reveals a Carotenoid Dark State in Purple Bacteria. Science340, 52-56 (2013).

36. Halpin, A. et al. Two-dimensional spectroscopy of a molecular dimer unveils the effects of vibronic coupling on exciton coherences. Nat Chem6, 196-201 (2014).

37. Tiwari, V., Peters, W.K. \& Jonas, D.M. Electronic resonance with anticorrelated pigment vibrations drives photosynthetic energy transfer outside the adiabatic framework. Proceedings of the National Academy of Sciences110, 1203-1208 (2013).

38. Lazonder, K., Pshenichnikov, M.S. \& Wiersma, D.A. Easy Interpretation of Optical TwoDimensional Correlation Spectra. Opt.Lett.31, 3354-3356 (2006).

39. Panitchayangkoon, G. et al. Long-lived quantum coherence in photosynthetic complexes at physiological temperature. Proceedings of the National Academy of Sciences107, 1276612770 (2010).

40. Bayliss, S.L. et al. Geminate and Nongeminate Recombination of Triplet Excitons Formed by Singlet Fission. Physical Review Letters112, 238701 (2014).

41. Wilson, M.W.B., Rao, A., Ehrler, B. \& Friend, R.H. Singlet Exciton Fission in Polycrystalline Pentacene: From Photophysics toward Devices. Accounts Chem. Res.46, 1330-1338 (2013).

42. Rao, A. et al. Exciton fission and charge generation via triplet excitons in pentacene/C60 bilayers. Journal of the American Chemical Society (2010).

43. Egorova, D. Oscillations in two-dimensional photon-echo signals of excitonic and vibronic systems: Stick-spectrum analysis and its computational verification. The Journal of Chemical Physics140, - (2014).

44. De Boeij, W.P., Pshenichnikov, M.S. \& Wiersma, D.A. System-bath correlation function probed by conventional and time-gated stimulated photon echo. Journal of Physical Chemistry100, 11806-11823 (1996).

45. Song, Y., Hellmann, C., Stingelin, N. \& Scholes, G.D. The separation of vibrational coherence from ground- and excited-electronic states in P3HT film. The Journal of Chemical Physics142, 212410 (2015).

46. Schlau-Cohen, G.S. et al. Elucidation of the timescales and origins of quantum electronic coherence in LHCII. Nat Chem4, 389-395 (2012). 
47. Egorova, D. \& Domcke, W. Coherent vibrational dynamics during ultrafast photoinduced electron-transfer reactions: quantum dynamical simulations within multilevel Redfield theory. Chemical Physics Letters384, 157-164 (2004).

48. Chan, W.-L., Tritsch, J.R. \& Zhu, X.Y. Harvesting Singlet Fission for Solar Energy Conversion: One- versus Two-Electron Transfer from the Quantum Mechanical Superposition. Journal of the American Chemical Society134, 18295-18302 (2012).

49. Falke, S.M. et al. Coherent ultrafast charge transfer in an organic photovoltaic blend. Science344, 1001-1005 (2014).

50. Romero, E. et al. Quantum coherence in photosynthesis for efficient solar-energy conversion. Nat Phys10, 676-682 (2014). 


\title{
Supplementary Information for: Real-Time Observation of Multiexcitonic States in Ultrafast Singlet Fission Using Coherent 2D Electronic Spectroscopy
}

\author{
Artem A. Bakulin ${ }^{1,2}$, Sarah E. Morgan ${ }^{2}$, Tom B. Kehoe ${ }^{2}$, Mark W.B Wilson ${ }^{2}$, Alex W. Chin ${ }^{2}$, Donatas \\ Zigmantas $^{4}$, Dassia Egorova*3, and Akshay Rao*2 \\ ${ }^{1}$ FOM Institute AMOLF, 1098 XG Amsterdam, The Netherlands \\ ${ }^{2}$ Cavendish Laboratory, University of Cambridge, JJ Thomson Avenue, Cambridge CB30HE, UK \\ ${ }^{3}$ Institut für Physikalische Chemie, Christian-Albrechts-Universität zu Kiel, Olshausenstr. 40, D-24098 \\ Kiel, Germany \\ ${ }^{4}$ Department of Chemical Physics, Lund University, P.O. Box 124, 22100 Lund, Sweden \\ * Corresponding authors: \\ degorova@gmail.com, ar525@cam.ac.uk
}

\section{Contents}

S.1Theory

S.1.1 Coherences in the beating maps . . . . . . . . . . . . . . . . . . . . . . . 2

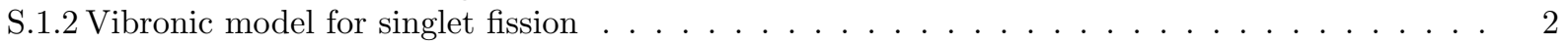

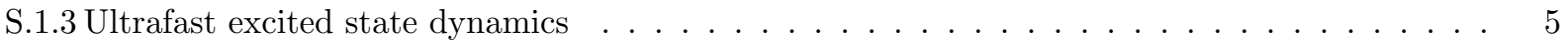

S.1.4 Simulation of Beating Maps . . . . . . . . . . . . . . . . . . . . . . 8

S.1.5 Beating Maps . . . . . . . . . . . . . . . . . . . . . . . . . . . 9

S.1.6 Distinguishing between GSB and excited state coherences . . . . . . . . . . . . . . 11

S.1.6.1 Rephasing . . . . . . . . . . . . . . . . . . . . . . . . . . . 12

S.1.6.2 Non-rephasing . . . . . . . . . . . . . . . . . . . . . . . 12

$\begin{array}{ll}\text { S.2Experiment } & 14\end{array}$

S.2.12D spectroscopy setup . . . . . . . . . . . . . . . . . . . . . . . . . 14

S.2.2 Absorptive 2D spectra of TIPS . . . . . . . . . . . . . . . . . . . . . 15

S.2.3 Absorptive 2D spectra of DTP . . . . . . . . . . . . . . . . . . . . . . 15

S.2.4 $2 \mathrm{D}$ decay associated spectra of pentacene . . . . . . . . . . . . . . . . 16

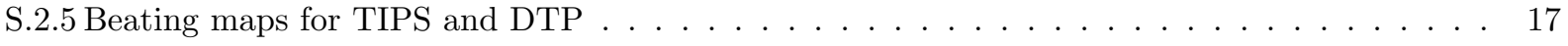

S.2.6 Refractive wings of peaks in $2 \mathrm{D}$ spectra . . . . . . . . . . . . . . . . . . . . 17

S.2.7 $2 \mathrm{D}$ response at high photon energy excitation/probe (from 2 to $2.4 \mathrm{eV})$. . . . . . . . . . . 19

S.2.8 Beating maps for the high photon energy excitation data . . . . . . . . . . . . . . . . . 21

S.2.9 Cross-polarisation data . . . . . . . . . . . . . . . . . . . . . . . . . . 23 


\section{S.1 Theory}

\section{S.1.1 Coherences in the beating maps}

Excitation of the Raman-active modes has a large impact on the coherent signatures in the signals. On the one hand, these modes can influence and even drive ultrafast fission dynamics in the photo-excited state, on the other hand they lead to a significant GSB contribution (ground-state coherence) to the recorded beating maps. Below we introduce a model which allows us to rationalise the peaks observed at the excitation frequency of about $14800 \mathrm{~cm}^{-1}(1.83 \mathrm{eV})$ in all beating maps as a strong GSB contribution, while the peaks resolved at lower excitation frequencies of around $13580 \mathrm{~cm}^{-1}(1.72 \mathrm{eV})$ are shown to originate from excited state coherences and cannot be explained by GSB. The excitation frequency of $1.72 \mathrm{eV}$ corresponds to the location of the dark multiexcitonic state, and the created excited-state coherence enables its spectroscopic detection in the beating maps.

To better understand how the Raman modes help to resolve the multiexcitonic state as well as the role of vibronic effects in singlet fission, we construct a model that includes three Raman active modes of frequencies $265 \mathrm{~cm}^{-1}, 1170 \mathrm{~cm}^{-1}$ and $1360 \mathrm{~cm}^{-1}$. In the following, we first introduce the model and discuss its ultrafast coherent dynamics following laser excitation, and then report and explain the beating maps it produces.

\section{S.1.2 Vibronic model for singlet fission}

The only attempt to account for vibrational degrees of freedom in singlet fission is reported in Refs. [1, 2], where time-dependent populations of electronic states weakly coupled to a harmonic bath are presented. Here a strong coupling between the electronic and vibrational degrees of freedom is in focus, and the vibrational modes are treated explicitly.

Our choice of the relevant Raman modes is motivated by experimental observations. As for the electronic basis, we adopt a minimal description that includes only two nonradiatively coupled states, one singlet state and the so-called multiexciton triplet state. The singlet state, $\left|S_{1}\right\rangle$, is already delocalised (i.e., it is a superposition of singlet excitations on several coupled pentacene molecules). This optically bright state is a singly excited state and the lowest in energy as compared to other delocalised singlets. The multiexciton triplet state, $|T T\rangle$, corresponds to an entangled superposition of two triplet excitations with opposite spins, correlated such that they form a spin-singlet pair (the total spin is zero). This state, although spin-allowed, is optically dark due to its doubly excited (2-electron) character. In order to enable a radiationless transition between the two electronic states, an interstate coupling $J$ is introduced.

There has been considerable effort to theoretically characterize singlet fission in pentacene in terms of electronic structure $[3,4,5,6]$. The nature of the coupling between the singlet and multiexciton triplet excitations has been widely discussed $[7,8,1,9,10,11,12,3,13,14,15,4,5$, 6], and proposed mechanisms include the 'direct' two-body coulomb coupling $[12,14]$ and the presence of an intermediate double triplet state which is degenerate with the singlet exciton $[9,16,10]$. Recent studies indicate that the 'indirect' coupling, mediated by the mixing of the singlet and triplet excitations with charge-transfer (CT) excitons dominates the coupling, leading to reported values in the range $200-900 \mathrm{~cm}^{-1}[7,8,1,3]$. There is some debate over the energies of the CT states and whether they participate as actual intermediates in the SF process, or as virtual states that mediate effective coupling of $S_{1}$ and $T T$ via a type of 'super-exchange' $[7,8,1,11,12,3]$. Here, we rather assume the latter view and believe that the sole effect of CT states is to generate a highly conservative coupling of $J=248 \mathrm{~cm}^{-1}$ between $S_{1}$ and $T T$ states (whose implicit, partial CT character will not be explicity referenced in the following).

To simulate the 2D PE signal, we also need to include the electronic ground states, $\left|S_{0}\right\rangle$, as well as a higher lying state to account for ESA. The experimentally determined transition energy of the ESA process corresponds to the $|T\rangle \rightarrow\left|T_{n}\right\rangle$ transition of pentacene. We thus denote the higher-lying state as $\left|T T_{n}\right\rangle$ and believe that this state represents a superposition of one single triplet excitation $(\rightarrow|T\rangle)$ and one double triplet excitation $\left(\rightarrow|T\rangle \rightarrow\left|T_{n}\right\rangle\right)$. Optical transitions are possible, therefore, between $\left|S_{0}\right\rangle$ and $\left|S_{1}\right\rangle$ states, as well as between $|T T\rangle$ and $\left|T T_{n}\right\rangle$. The interaction with the light field is given, in the electric dipole approximation, 
by $H_{\text {int }}=-\mu E(t)$ where $E(t)$ is the time-dependent electric field of the applied pulse sequence and $\mu$ is the electric transition dipole operator. In the introduced electronic basis the dipole operator reads

$$
\mu=\mu_{S_{0}, S_{1}}|S\rangle\left\langle S_{0}\left|+\mu_{T T, T T_{n}}\right| T T\right\rangle\left\langle T T_{n}\right|+H . c .,
$$

where the transition dipoles moments for $S_{0} \rightarrow S_{1}$ and $T T \rightarrow T T_{n}$ are denoted by $\mu_{S_{0} S_{1}}$ and $\mu_{T T, T T_{n}}$, respectively. Following experimental and theoretical studies of the strong triplet excited state absorption, we take $\mu_{T T, T T_{n}}=2.5 \mu_{S_{0} S_{1}}[17,18]$.

The system Hamiltonian reads

$$
H=\sum_{i \in\left\{S_{0}, S_{1}, T T, T T_{n}\right\}}|i\rangle h_{i}+\epsilon_{i}\langle i|+\left(\left|S_{1}\right\rangle J\langle T T|+H . c .\right),
$$

where $\epsilon_{i}$ denote the vertical excitation energies (zero in the ground state), and the vibrational Hamiltonians pertaining to each electronic state, $h_{i}$, are assumed to be harmonic and given by

$$
h_{i}=\sum_{k} \omega_{k}\left(a_{k}^{\dagger} a_{k}+\frac{1}{2}\right)-\omega_{k} \frac{\Delta_{k}^{i}}{\sqrt{2}}\left(a_{k}+a_{k}^{\dagger}\right) .
$$

The coupling of each mode to the excited electronic states is quantified by the dimensionless displacement with respect to the ground-state geometry, $\Delta_{k}^{i}\left(\Delta_{k}^{S_{0}} \equiv 0\right)$. For the $\left|S_{1}\right\rangle$ state, we make use of the values obtained by Gisslen et al. using density functional theory $\left(\Delta_{1}^{S_{1}}=0.7, \Delta_{2}^{S_{1}}=0.6, \Delta_{3}^{S_{1}}=0.75\right)[19]$. No information on the geometry of the $|T T\rangle$ and $\left|T T_{n}\right\rangle$ states is - to our knowledge - available in the literature. In the following simulations, we chose $\Delta_{k}^{T T}=0$ and $\Delta_{k}^{T T_{n}}=\Delta_{k}^{S_{1}}$. This choice is motivated by experimental observations, since it results in a very good agreement between the simulated and experimental beating maps (see subsection S.1.4 below).

Diagonalising the system Hamiltonian, Eq. (S.2), to obtain the eigenstates $\left|\phi_{n}\right\rangle$ of energy $E_{n}$, we vary the vertical energies of the electronic states so that the lowest energy eigenstate corresponds to the measured excitation energy of the $|T T\rangle$ state $(1.72 \mathrm{eV})$ and the first strongly absorbing state, as quantified by the transition dipole moment from the ground state (vide infra), matches the measured singlet exciton energy $(1.83 \mathrm{eV})$. This results in $\epsilon_{S_{1}}=15412 \mathrm{~cm}^{-1}$ and $\epsilon_{T T}=13910 \mathrm{~cm}^{-1}$. To fit the $2 D$ spectra, we take $\epsilon_{T T_{n}}=$ $29478 \mathrm{~cm}^{-1}$.

The eigenstates of the Hamiltonian (S.2) can be divided into three distinct manifolds. The ground state manifold $|\mathbf{g}\rangle$ consisting of the vibrational states, as well as a higher excited manifold, $|\mathbf{f}\rangle$, which represents the vibrational (vibronic) manifold of the $\left|T T_{n}\right\rangle$ state, are not influenced by the coupling $J$. These two manifolds are introduced to account for spectroscopic transitions relevant for the 2D signal, they are not immediately involved in the fission process. The crucial manifold for the ultrafast excited-state dynamics is the excited manifold $|\mathbf{e}\rangle$ : its states are superpositions of the excited electronic basis states, namely the $\left|S_{1}\right\rangle$ and $|T T\rangle$ states and their vibrational excitations, see Supplementary Fig. S1. They can be directly excited from the ground state by the laser (the $|\mathbf{f}\rangle$-states can only be excited from $|\mathbf{e}\rangle$, i.e. not directly from the ground state). Note that due to $\left|S_{1}\right\rangle$ contributions, all transitions from the initial state to the eigenstates of $|\mathbf{e}\rangle$ are optically allowed, although only states with a significant $\left|S_{1}\right\rangle$ superposition weight have appreciable transition dipole moments to the ground state.

This is illustrated in Supplementary Fig. S2a, which shows the computed transition dipole moments for excitation from the electronic and vibrational ground state of the relevant lowest energy eigenstates in $|\mathbf{e}\rangle$ as a function of excitation energy (eigenstates are numbered upwards from the lowest energy state) and the fraction of $\left|S_{1}\right\rangle$ contributing to each eigenstate.

The strongest transition corresponds to eigenstate 5, which has $86 \%$ singlet character and occurs at the energy of the dominant absorption features in the $2 D$ spectra $\left(14750 \mathrm{~cm}^{-1}, 1.83 \mathrm{eV}\right)$. The lowest energy state, state 1, which has over $95 \%|T T\rangle$ character, has a dipole moment approximately three times smaller. This "borrowed" dipole moment arises from the weak electronic admixture of the bright $\left|S_{1}\right\rangle$ state in this 


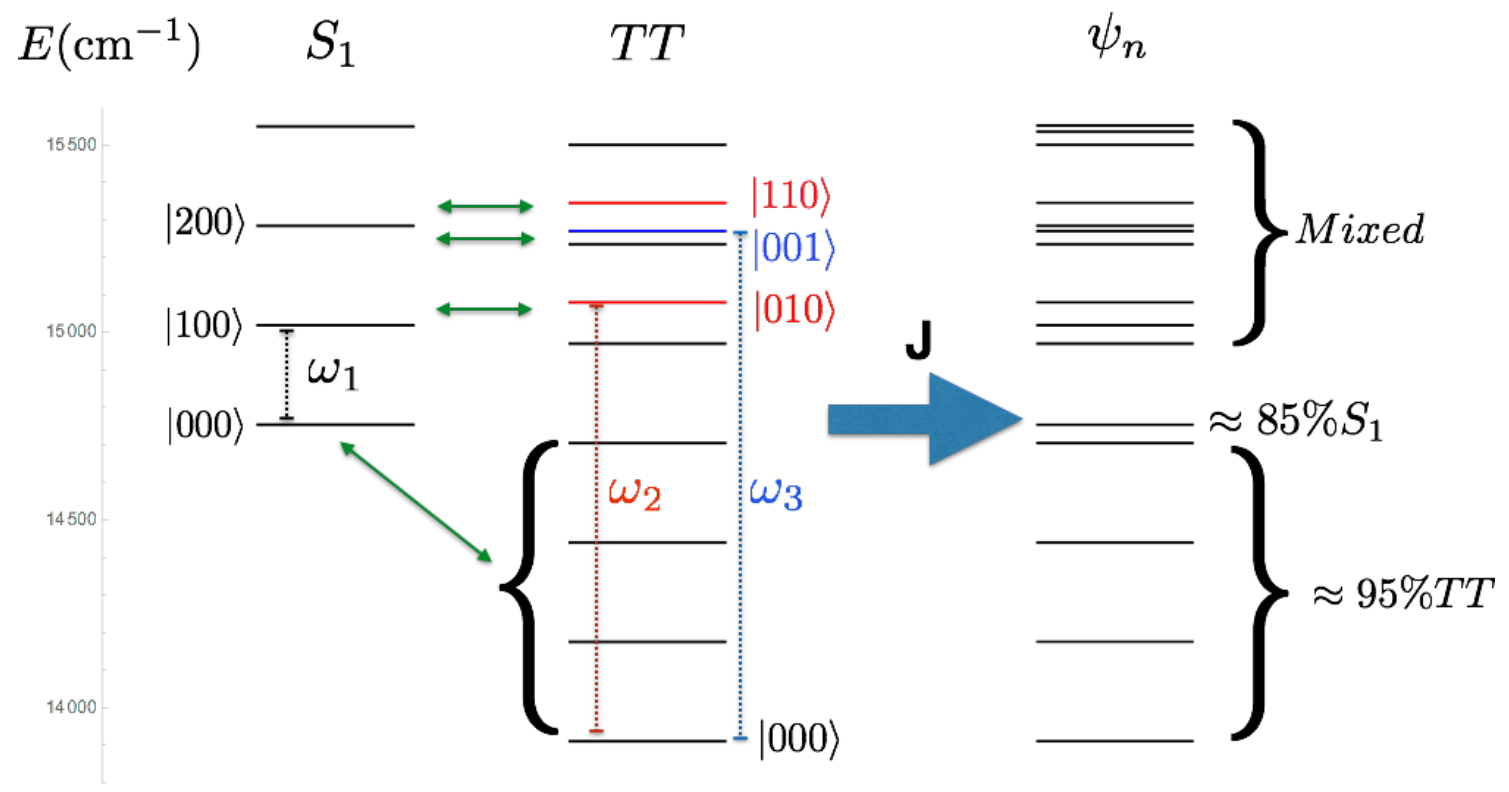

Supplementary Figure S1: (Left) The eigenstates of the excited state manifold $|\mathbf{e}\rangle$ of our model without electronic coupling $(J=0)$, showing the energetic alignment of the vibrational levels on the $\left|S_{1}\right\rangle$ and $|T T\rangle$ excited states. The vibrational excitations of $|T T\rangle$ (shown in red and blue) lie close in energy to a number of excited states on $\left|S_{1}\right\rangle$. The vibrational states are labelled by the occupation numbers as $\left|n_{1}, n_{2}, n_{3}\right\rangle$ where $n_{1}, n_{2} \& n_{3}$ are the numbers of quanta in modes of frequency $\omega_{1}=265 \mathrm{~cm}^{-1}, \omega_{2}=1170 \mathrm{~cm}^{-1} \& \omega_{3}=$ $1360 \mathrm{~cm}^{-1}$, respectively. (Right) The emerging eigenstates of $|\mathbf{e}\rangle\left(\psi_{n}\right)$ when electronic coupling is switched on $\left(J=248 \mathrm{~cm}^{-1}\right)$. The states are superpositions of $\left|S_{1}\right\rangle,|T T\rangle$ and their vibrational excitations, the major electronic contributions of some of the states are shown as percentages. As a result of the closeness in energy of several of the vibrationally excited states for $J=0$, strongly mixed character can appear amongst the eigenstates at energies slightly above that of the orginal, uncoupled $\left|S_{1}\right\rangle$ state for finite $J$. 

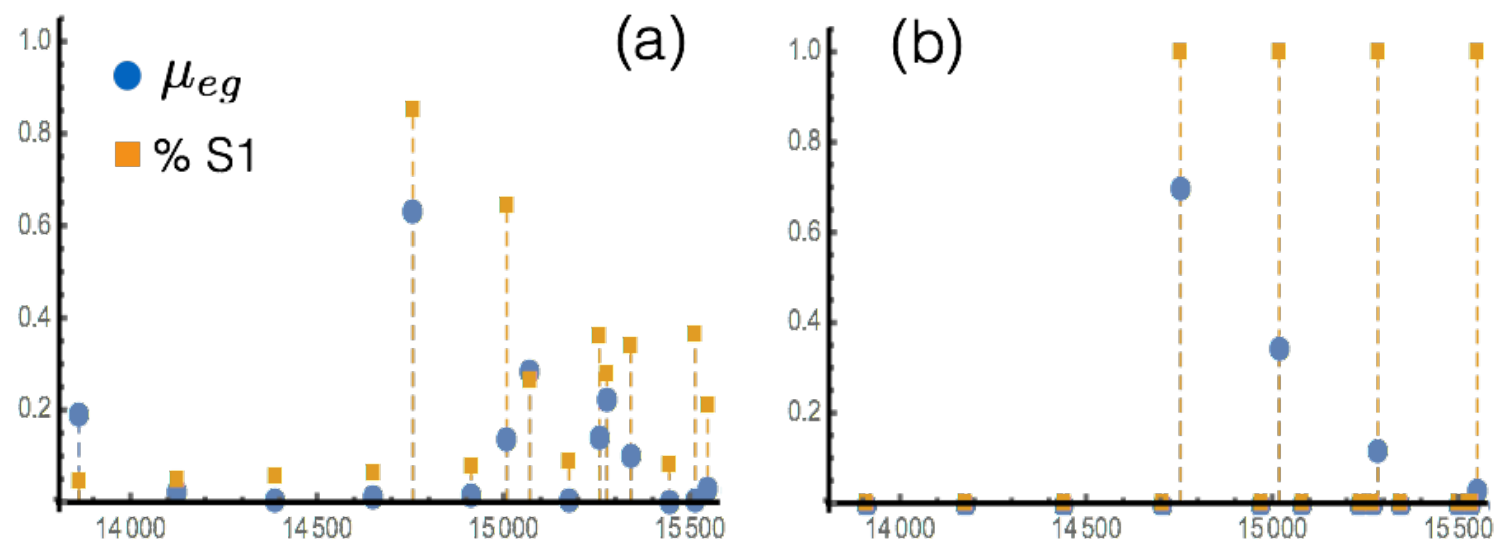

Supplementary Figure S2: (a) Dipole moments (circles) and fraction of $S_{1}$ (squares) contributing to the ten lowest eigenstates of our model. (b) The same as (a) for a model without electronic coupling, showing single vibrational progression $\left(265 \mathrm{~cm}^{-1}\right.$ mode) of the singlet exciton state. This structure is significantly altered in the presence of electronic couplings.

eigenstate. Note that less than $5 \%$ of the $\left|S_{1}\right\rangle$ character happens to be enough to detect the transition in the beating maps, while it is too low for detection by linear techniques: the relation between the transition intensities of the states 1 and 5 is 1:10 in the linear absorption spectrum, leaving the lowest state completely obscured in the tail of the much brighter transitions. However, as discussed in the main text and as shown below, non-linear signals involving this weak dipole moment in conjunction with stronger processes, notably the excited state absorption $|T T\rangle \rightarrow\left|T T_{n}\right\rangle$, allow for their detection in the 2D beating maps.

Supplementary Figure S2b shows the same quantities as Supplementary Fig. S2a with zero electronic coupling. Comparing these figures at energies above the main singlet transition, we see that the simple vibrational progression of the uncoupled singlet states (corresponding to sidebands of the $265 \mathrm{~cm}^{-1}$ mode in this energy range) becomes significantly distorted in the presence of coupling. In particular, eigenstates which contain appreciable contributions from the vibrationally excited $|T T\rangle$ states with one vibrational quantum in either the $1170 \mathrm{~cm}^{-1}$ and $1360 \mathrm{~cm}^{-1}$ modes (eigenstates 8 and 11, respectively), exhibit an efficient dipole borrowing. Note that the energy of these states is distorted only slightly as compared to the nonperturbed case $J=0$.

\section{S.1.3 Ultrafast excited state dynamics}

Before turning to the simulations of the 2D beating maps, let us briefly discuss the ultrafast dynamics of the introduced model following a laser excitation by a pulse with a Gaussian time envelope of 13 fs duration at FWHM and carrier frequency of $1.8 \mathrm{eV}$. In order to be able to describe the fast and irreversible decay of the singlet state (the experimentally estimated decay rate is about $80 \mathrm{fs}$ ), we extend the model and introduce 


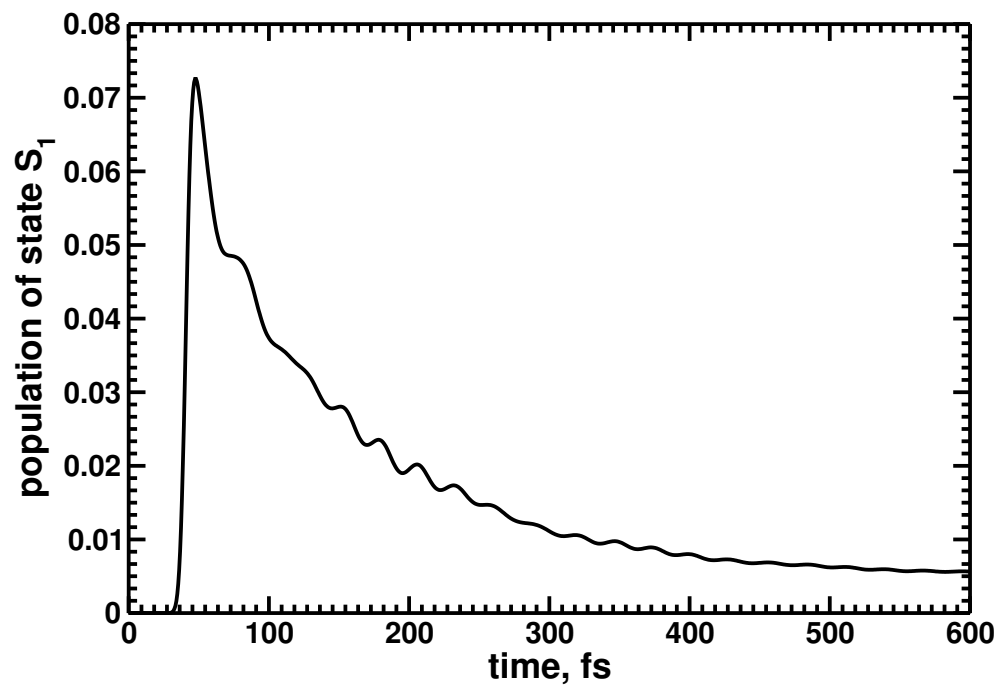

Supplementary Figure S3: Population of the $S_{1}$ state following laser excitation by a $13 \mathrm{fs}$ Gaussian pulse. Overall population decay into the low enegy triplet pairs, ignoring oscillatory features, is well described by a fission time constant of $83 \mathrm{fs}$, which is consistent with this and previous experiments.

a weak linear coupling to a harmonic bath described within multilevel Redfield approach [20]. The bath is characterized by the Ohmic spectral function $J(\omega)=\eta \exp \left(-\omega / \omega_{c}\right)$ with $\eta=0.3$ and $\omega_{c}=800 \mathrm{~cm}^{-1}$ and couples to the vibrational modes in our model as described in [20]. Simulations were carried out at room temperature with the system initially in the electronic ground state and a thermal equilibirum distribution of vibrational states. Supplementary Figure S3 shows the population of the optically bright $\left|S_{1}\right\rangle$ electronic state. The chosen relaxation parameters lead to the decay of the population at the experimentally observed timescale. Weak oscillations indicate that coherent motion has been created in the excited state. Which state pairs contribute mostly to the observed coherences is represented in Supplementary Fig. S4. Interestingly, these are mostly states with a significant contribution from the dark $|T T\rangle$ state. The interaction (mixing) between the $\left|S_{1}\right\rangle$ and $|T T\rangle$ states results in nonzero dipole moments between the initial state and the eigenstates which have a strong $|T T\rangle$ component (states 1-4, 6, 8, 9, 11 in Supplementary Fig. S2). The most pronounced coherence is observed between the lowest excited eigenstate (state 1, which has 95\% contribution from the vibrationally ground state of the $|T T\rangle$ state) and the eigenstates 2, 8 and 11 . These states have strong contributions from the vibrationally excited $|T T\rangle$ states: one vibrational quantum in $265 \mathrm{~cm}^{-1}$ (95\% of state 2$)$, in $1170 \mathrm{~cm}^{-1}$ mode ( $68 \%$ of state 8$)$, and in the $1360 \mathrm{~cm}^{-1}$ mode $(65 \%$ of state 11). Although all the three coherences have very similar amplitudes and characteristic dephasing timescales, the high-frequency oscillations shall be better resolved spectroscopically due to a larger admixture of the $\left|S_{1}\right\rangle$ state to states 8 and 11, and, therefore, stronger dipoles (cf. Supplementary Fig. S2).

The most pronounced and long-lived coherences are, therefore, between the states dominated by the same electronic character $(T T)$ and predominantly of vibrational origin.

The population dynamics of the eigenstates are shown in Supplementary Fig. S5. The three lowest states dominated by the $|T T\rangle$ character are shown by black lines. The population of the state with the strongest dipole transition from the initial state, state 5, is shown in red and essentially tracks the decay of the main singlet exciton from which fission proceeds. The fission dynamics are dominated by the population transfer from state 5 to the three lowest states. The populations of states 8 and 11 (blue and green lines, respectively), which contribute to the excited-state coherence detected in the maps of $1170 \mathrm{~cm}^{-1}$ and $1360 \mathrm{~cm}^{-1}$ modes, decay more slowly.

Interestingly, the observed eigenstate population dynamics of the model system allows us to propose an alternative interpretation of the near-instantanous 'hot' triplet signal observed by Chan et al. in the timeresolved two-photon photoemission studies of singlet fission in pentacene [9]. This signal was assigned by 


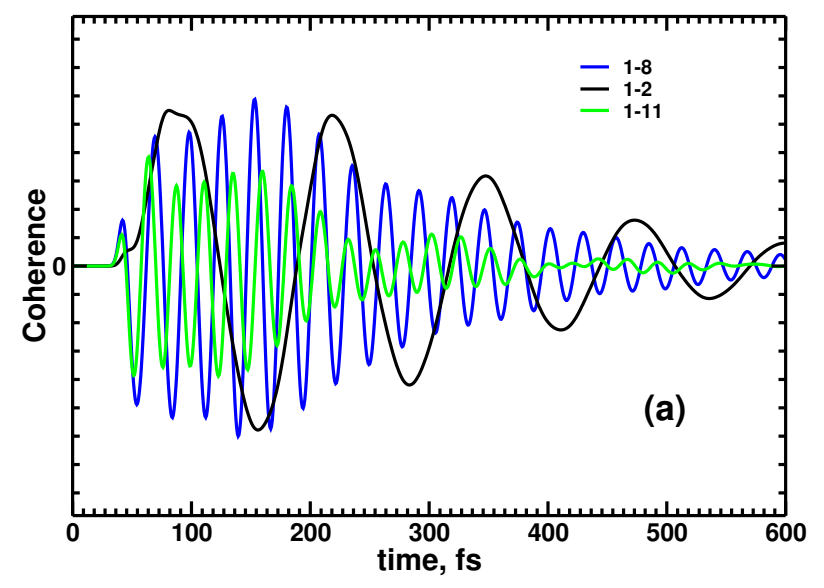

Supplementary Figure S4: Coherences between selected eigenstates of the model (see accompanying text).
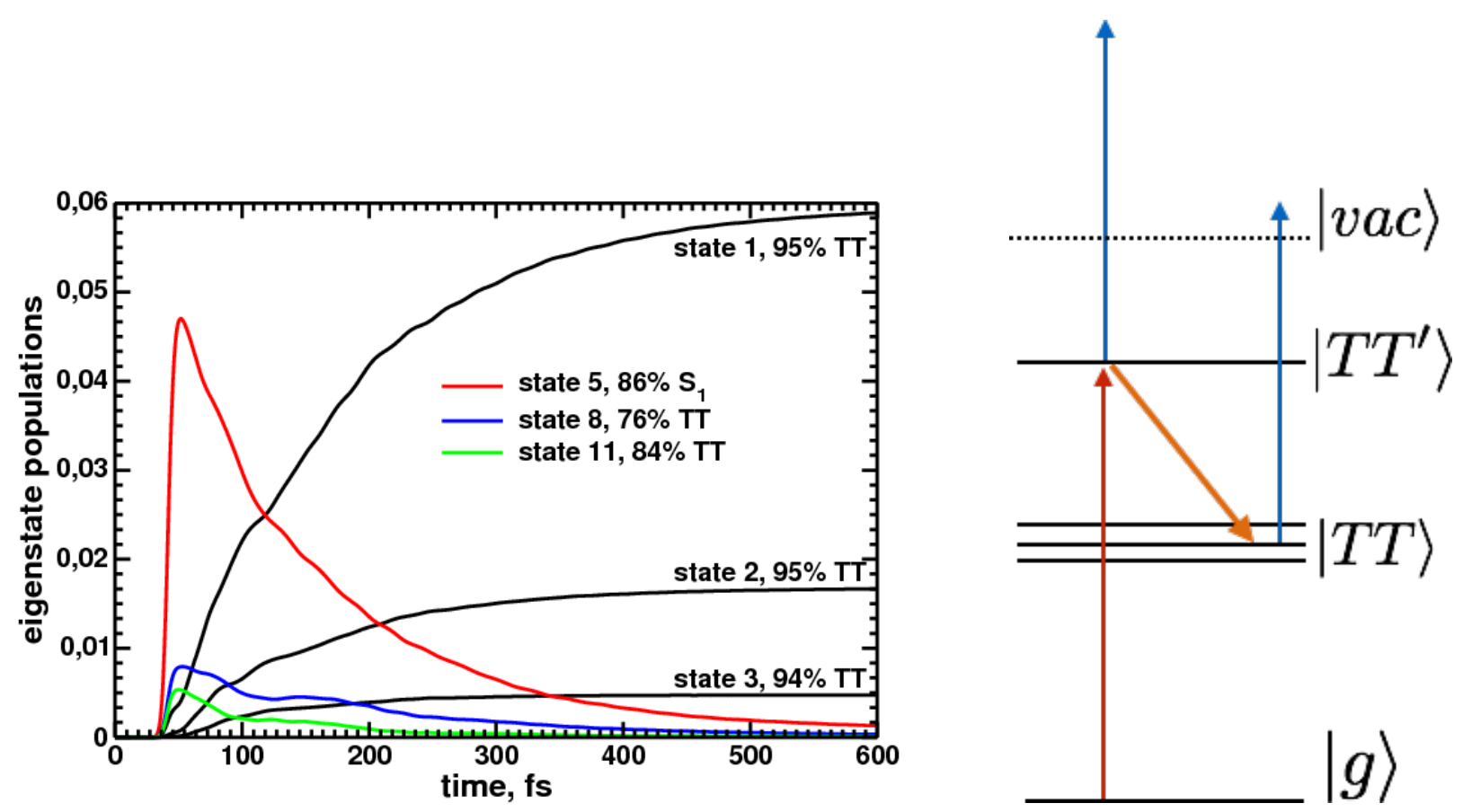

Supplementary Figure S5: (Left) Populations of selected eigenstates during singlet fission. (Right) Electronic coupling creates optically active eigenstates with significant contributions from triplet states containing vibrational quanta in the $1170 \mathrm{~cm}^{-1} \& 1360 \mathrm{~cm}^{-1}$ modes (such as states 8 and 11 on the left). These are directly excited by the pump laser (red arrow) and may be ionised at early times by a second high-energy laser pulse (blue arrow). As these higher energy states decay towards the lowest energy $|T T\rangle$ states (orange arrow), the energy of the emitted electrons may be expected to decrease, as observed by Chan et al. [9]. 
Chan et al. to a dark multiexciton state which was degenerate with the lowest enegy singlet transition and very strongly coupled to it $[9,10]$. Subsequent dephasing and relaxation processes, leading to the formation of free triplet pairs, were proposed as the origin of the shift (cooling) of the triplet signal to lower energies (by $0.11 \mathrm{eV}$ ) with a time constant of $\approx 200 \mathrm{fs}$. Our studies indicate that states $8(76 \%|T T\rangle)$ and 11 $(84 \%|T T\rangle)$ rise almost at the same rate as the main optical transition (state 5). These states of essentially vibrationally excited triplet character have energies close to the main optical transition $\left(200-500 \mathrm{~cm}^{-1}\right.$ maximum difference) and decay with relaxation rates which are significantly slower than the overall $80 \mathrm{fs}$ fission rate $(\approx 180 \mathrm{fs})$. We note that the decrease of the average energy of the triplet states orginating from these transitions is likely to be even slower, as relaxation may proceed through other, lower frequency, vibrational states on the way down to the lowest energy eigenstate. Putting these observations together, a possible interpretation of the results of [9] is sketched in Supplementary Fig. S5. Finally, we reiterate that in our model this process occurs alongside the ultrafast, direct decay of the main optical transition into the lower energy $|T T\rangle$-like states $(1-3)$ via environment-mediated dissipation, perhaps providing some insight into the range of fission timescales obtained in different types of experiment. Note also, that our interpretation suggests that the coupling to the multiexcitonic state $(J)$ does not need to be strong once vibrational motion is accounted for.

\section{S.1.4 Simulation of Beating Maps}

In the limit of infinitely short laser pulses and within a perturbative description of the light-matter interaction, any four-wave mixing signal, and photon echo in particular, can be expressed in terms of the third-order response functions [21]. The response functions can be conveniently decomposed into three physical processes, the ground state bleach (GSB), stimulated emission (SE) and excited state absorption (ESA), and schematically represented in terms of double-sided Feynman diagrams, as shown in Supplementary Fig. S6. So called rephasing and non-rephasing diagrams differ in the ordering of the first two laser pulses which define the coherence time. While conventional 2D photon echo signal implies Fourier transforming over the coherence time $\tau$ and rephasing time $t$, the beating maps are obtained by taking an additional Fourier transform over evolution time $T$.

The explicit form of the response functions is determined by the system's optical excitations and their dynamics in $\tau, T$, and $t$. In the absence of dissipative processes, the response functions are completely determined by the system's eigenstate structure and the transition dipole strengths between the individual levels. In the following we work in this so called stick-spectrum limit and exploit the Feynman diagram representation to simulate the beating maps, by calculating all possible Feynman diagrams which contribute to a given beating map.

We use simple Lorentzian lineshapes to account for the dephasing and relaxation rates, respectively of intereigenstate coherences and eigenstate populations during the $\tau, T \& t$ times. These are estimated from our earlier dissipative Redfield dynamics (effective rates are used in the Markovian limit assumed here). No additional effects due to population or coherence transfer are considered, although they are both present in the more advanced simulations of the dynamics in subsection S.1.3. In our simulations, all pathways involving transitions where the energy difference between the states falls outside of the laser spectrum were filtered out of the theoretical signal. For this filtering, a top-hat approximation to the power spectrum between $12950 \rightarrow 15800 \mathrm{~cm}^{-1}$ was employed. As a post-processing step, a Gaussian convolution is uniformly applied to the beating maps with a standard deviation of $75 \mathrm{~cm}^{-1}$, as a first and very simple approximation to the effect of static disorder (no other account is taken of inhomogeneous broadening and the related dynamical dephasing in $T$ ). With these approximations, our chosen method is not suited for the reproduction of subtle, highly transient and non-Markovian evolution of the spectra, where an advanced treatment of the open-system dynamics would be required. Fortunately, the striking features of the experimental beating maps are the positions of the peaks at each frequency and the stark differences between the rephasing and non-rephasing map patterns; as we now show, these are well described and explained by this simplified treatment. 

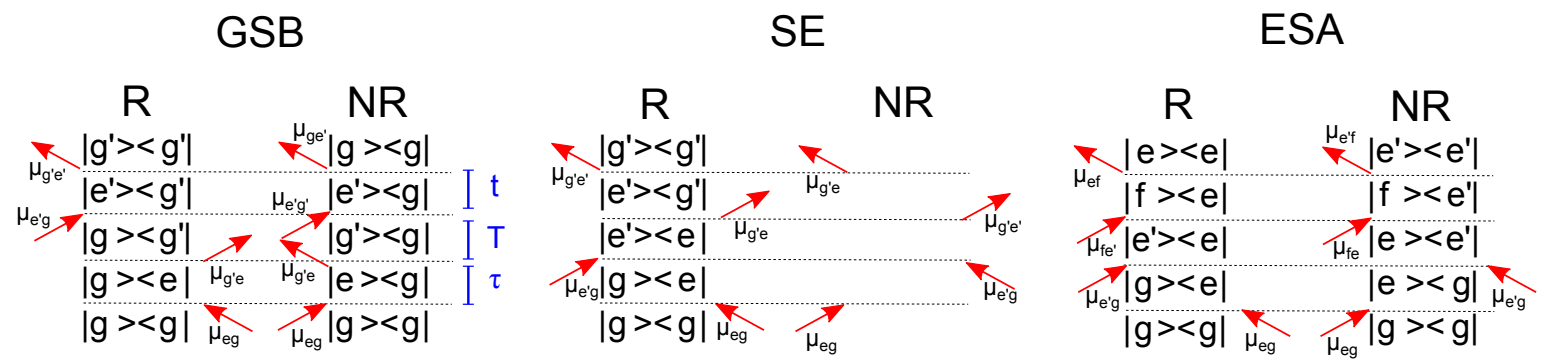

Supplementary Figure S6: Generic Feynman diagrams for the third-order nonlinear optical response functions. The coherence $(\tau)$, waiting $(T)$ and rephasing $(t)$ times are indicated, as are the relevant dipole moments $\mu_{i j}$ whose product determines the amplitude of a diagram's contribution to the signal. (a) Ground state bleach (GSB) diagrams involve dynamical evolution in the ground state manifold during the evolution time. (b) Stimulated emission (SE) involves excited state evolution in the evolution time and terminates in emission back to the ground state. (c) Excited state absorption (ESA) also involves excited state evolution in $T$ but terminates in a transition involving the doubly excited $|\mathbf{f}\rangle$ manifold.

\section{S.1.5 Beating Maps}

In Supplementary Fig. S7 experimental and simulated beating maps of the oscillations with frequencies $265 \mathrm{~cm}^{-1}, 1170 \mathrm{~cm}^{-1}$ and $1360 \mathrm{~cm}^{-1}$ are compared. The qualitative agreement with the experimental data is very good in terms of peak positions and relative intensities, and we reproduce the change in peak patterns between rephasing and non-rephasing maps with a single parameter set and close agreement with a recent set of general rules for 2D spectra given in Ref. [22]. We now discuss the main interrelations between the eigenstate spectrum and coherent dynamics described in subsections 1.2 and 1.3 and the structure seen in the beating maps.

The maps are dominated by essentially two contributions: GSB and ESA (the third, SE contribution to the oscillatory signal component is negligible due to the very short life time of the $S_{1}$ state). The GSB contribution represents the ground-state coherence and produces beating map signals at exactly the vibrational frequencies of the modes (excitation of the Raman modes in the ground electronic state). ESA arises due to excited-state coherent motion, but both ESA and GSB contributions can be found in the same maps, since coherences with frequencies very close to those of the ground-state vibrational modes are found in the excited state (see Supplementary Fig. S4).

Let us first identify the GSB contribution to the beating maps. Efficient Raman scattering is expected for the strongest transition at $\omega_{\mathrm{ex}}=1.83 \mathrm{eV}$ for all modes considered (cf. Fig. 3 in the main text). In the simulations, this state is of $86 \%$ singlet character (state 5), and the corresponding GSB pathway (with $|e\rangle=\left|e^{\prime}\right\rangle=\left|\psi_{5}\right\rangle$ in the GSB diagram, Supplementary Fig. S6) results in peak 1 in the non-rephasing (NR) maps, and in peaks 2a, 2b, 2c in the rephasing (RP) maps. State $|g\rangle$ in Supplementary Fig. S6 denotes the initial, vibrational ground state, and $\left|g^{\prime}\right\rangle$ corresponds to the excitation of one vibrational quantum (the system is in a vibrational coherence in the electronic ground state in the evolution time) in one of the modes considered. Peak 1 in the NR maps has the same location for all modes: its excitation and probe frequencies correspond to the transition at $1.83 \mathrm{eV}$. In the RP maps, the location of the GSB peak depends on the mode frequency $\Omega$ (see rephasing GSB diagram in Supplementary Fig. S6), the peaks $2 \mathrm{a}, 2 \mathrm{~b}, 2 \mathrm{c}$ are found at $\omega_{\mathrm{ex}}=1.83 \mathrm{eV}$ and $\omega_{\mathrm{pr}}=\omega_{\mathrm{ex}}-\Omega$, i.e., at $\omega_{\mathrm{pr}} \approx 1.8,1.68$ and $1.65 \mathrm{eV}$, respectively. Some additional GSB peaks are resolved at frequencies higher than $1.83 \mathrm{eV}$, they can be explained as Raman process involving the state 7 , these finer features on the edge of the experimental bandwidth will be explored elsewhere.

The GSB contribution would have been detected even in the absence of the coupling between singlet and multiexciton triplet states. As for the ESA contribution, it arises due to triplet-triplet absorption and represents, therefore, the probe of the fission process. Its coherent (oscillatory) component reflects the excited-state coherences. In the simulations these coherences are between the states of primarly $|T T\rangle$ character (1-2, 1-8 


\section{Experiment Modeling}

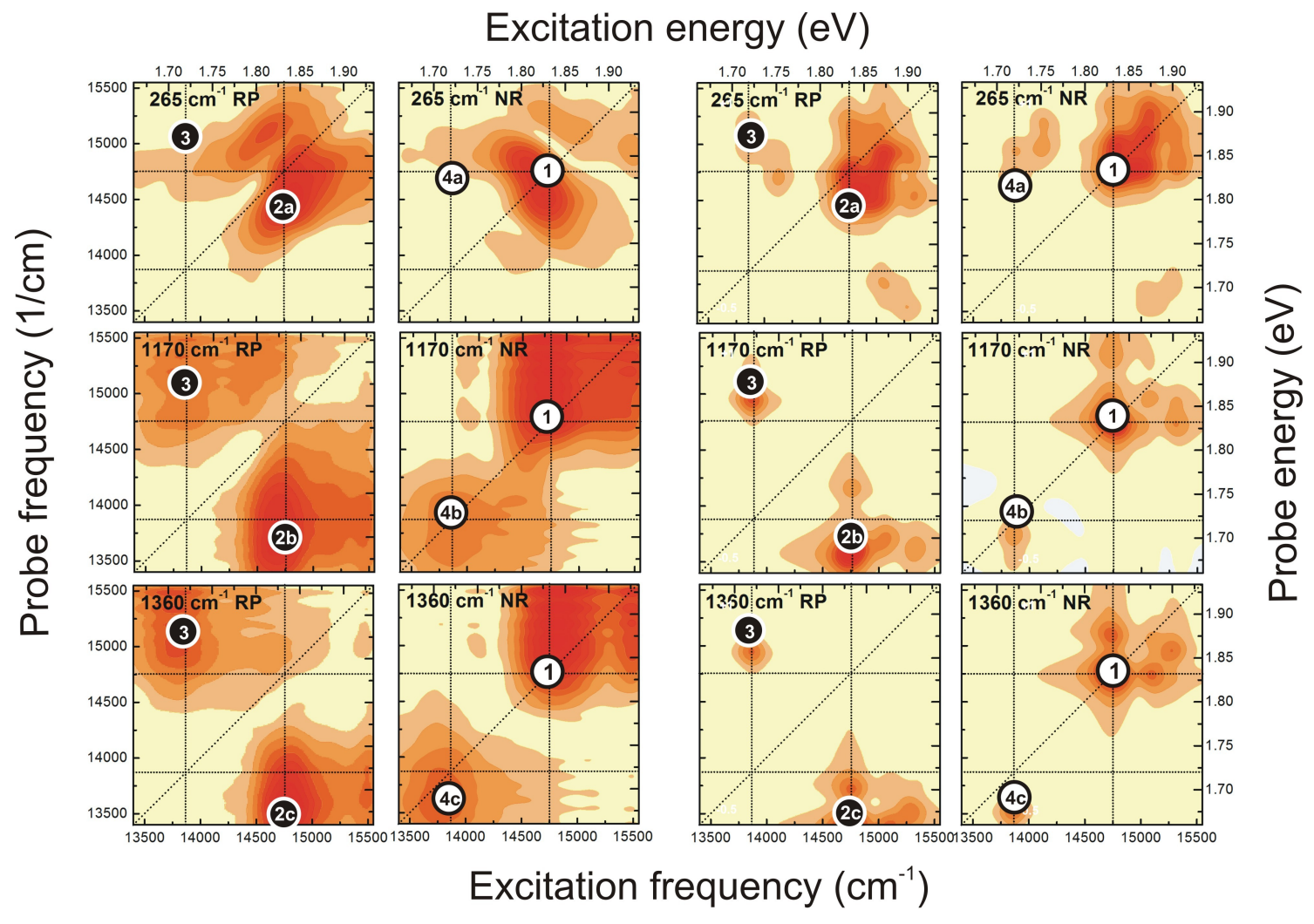

Supplementary Figure S7: Experimental and simulated beating maps of modes $265 \mathrm{~cm}^{-1}, 1170 \mathrm{~cm}^{-1}$ and $1360 \mathrm{~cm}^{-1}$. 
and 1-11, Supplementary Fig. S4) and beat at frequencies very close to that of the vibrational modes, that is why ESA and GSB contributions are found in the same maps. The peaks 3, 4a, 4b, and 4c in Supplementary Fig. S7 arise from ESA diagrams (Supplementary Figure S6) with $|e\rangle=\left|\psi_{1}\right\rangle,\left|e^{\prime}\right\rangle=\left|\psi_{2,8,11}\right\rangle$. The excitation frequency of these peaks corresponds to the very weak transition at $\omega_{\mathrm{ex}}=1.72 \mathrm{eV}$. This absorption frequency is expected for the multiexciton $|T T\rangle$ state, but the transition has never been directly resolved experimentally. Its resolution in the beating maps becomes possible due to very strong ESA process from the $|T T\rangle$ state to $\left|T T_{n}\right\rangle$ state. In our model, the transition at $1.72 \mathrm{eV}$ corresponds to the lowest eigenstate $\left|\psi_{1}\right\rangle$, it is of $95 \%|T T\rangle$ character. For this state, the transition dipole from the ground state is nonzero due to the weak $S$-state contribution, but too weak to resolve the transition in the linear absorption. In the beating maps, the weakness of this transition is compensated by very strong ESA dipoles to the higher triplet states. The probe frequency $\omega_{\text {pr }}$ of ESA peaks is determined by the location of the absorbing $\left|T T_{n}\right\rangle$ state. As predicted by Feynman diagrams, it is the same in the rephasing maps of all modes (peak $3, \omega_{\mathrm{pr}}=1.88 \mathrm{eV}$ ), but it is lowered by the mode frequency $\Omega$ in the NR maps (peaks $4 \mathrm{a}, 4 \mathrm{~b}$, and 4c) as compared to the $\omega_{\text {pr }}$ of peak 3 . This predicted redshift shift of the probe frequency agrees very well with the experimental data, particularly in the $1170 \mathrm{~cm}^{-1}$ and $1360 \mathrm{~cm}^{-1}$ maps. Note, that although the amplitudes of coherences $1-2,1-8$ and $1-11$ in Supplementary Fig. S4 are alike, the intensity of ESA peaks in the map of the $265 \mathrm{~cm}^{-1}$ mode is much weaker than that of the high-frequency modes. The reason is that the transition dipoles from the ground state are much stronger for the states $\left|\psi_{8}\right\rangle$ and $\left|\psi_{11}\right\rangle$ as compared to the state $\left|\psi_{2}\right\rangle$ (Supplementary Figure S2a).

\section{S.1.6 Distinguishing between GSB and excited state coherences}

During the evolution time, T, oscillations can occur with either positive or negative frequencies. Here we show that by inspecting the sign of the oscillation frequency, we can assign whether the peaks correspond to signals which evolve on the ground or excited state during the evolution time. This method has already been proposed and exploited in the literature [23, 24, 25].

In order to distinguish whether the sign of the oscillation frequency at a particular peak position is positive or negative, we take the Fourier transform of the complex valued data over the evolution time, T, according to:

$$
\hat{f}\left(\omega_{T}\right)=\int_{-\infty}^{+\infty} f(T) e^{-i 2 \pi \omega_{T} T} d T
$$

where $f(T)$ is the complex valued signal at a specific point in the $2 \mathrm{D}$ spectrum (note that throughout the rest of this work the Fourier transform is taken over the real valued data). Using the complex valued data allows us to distinguish between positive and negative oscillation frequencies. In the Feynman diagrams, a positive frequency means that during the evolution time the state on the 'ket' side is at a lower energy than the state on the 'bra' side (e.g. $|g\rangle\left\langle g^{\prime}\right|$, where' denotes the vibration) and vice-versa for a negative frequency (e.g. $\left.\left|g^{\prime}\right\rangle\langle g|\right)[23]$.

We consider four specific points in the $2 \mathrm{D}$ spectrum, which correspond to the positions of the four strongest peaks in the $\omega_{T}=1360 \mathrm{~cm}^{-1}$ beating maps, namely the points $\left(\omega_{\tau}, \omega_{t}\right)=(13870,15015) \mathrm{cm}^{-1}$ and $(14765,13401) \mathrm{cm}^{-1}$ in the rephasing spectrum and $(14765,14762) \mathrm{cm}^{-1}$ and $(13870,13655) \mathrm{cm}^{-1}$ in the non-rephasing spectrum. The $1360 \mathrm{~cm}^{-1}$ beating maps are shown for convenience in Supplementary Fig. S8, with the points discussed above labelled from 1-4. The Fourier transforms of the complex valued 2D signal at these points over the evolution time are shown in Supplementary Fig. S9.

Note that the Fourier transforms of the complex valued signals at the positions corresponding to the peaks in the $1170 \mathrm{~cm}^{-1}$ beating map show exactly the same pattern of peaks at $1170 \mathrm{~cm}^{-1}$ as we observe in Supplementary Fig. S9 at $1360 \mathrm{~cm}^{-1}$. This is expected since the origins of the peaks (GSB and ESA) are

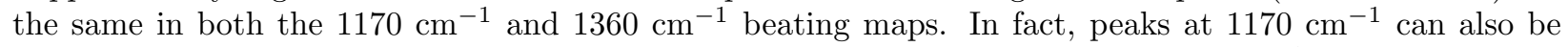
observed in Supplementary Fig. S9 due to the close proximity of the 1170 and $1360 \mathrm{~cm}^{-1}$ peaks. 


\section{S.1.6.1 Rephasing}

From the Fourier transforms in Supplementary Fig. S9, the rephasing upper cross-peak has a prominent peak at $-1360 \mathrm{~cm}^{-1}$ in the Fourier transform, whilst the rephasing lower cross-peak has a prominent peak at $+1360 \mathrm{~cm}^{-1}$.

The Feynman diagrams for the peaks are shown in Supplementary Fig. S10. As observed experimentally, the sign of the oscillations is negative for the upper cross-peak (because the 'ket' state has a higher energy than the 'bra' state) and positive for the lower cross-peak. Note that if the upper cross-peak came from GSB, the sign of the oscillations would be positive and we would also see a peak in the Fourier transform at $+1360 \mathrm{~cm}^{-1}$. The absence of this peak means that we can unambiguously assign this signal to excited state coherences and rule out the possibility of significant GSB.

\section{S.1.6.2 Non-rephasing}

Similarly, the non-rephasing upper diagonal peak has a prominent peak at $-1360 \mathrm{~cm}^{-1}$ in the Fourier transform. Meanwhile the non-rephasing lower (near) diagonal peak has a prominent peak at $+1360 \mathrm{~cm}^{-1}$. From the Feynman diagrams in Supplementary Fig. S10, the sign of the oscillations is negative for the upper diagonal peak and positive for the lower diagonal peak, as observed experimentally. This is inline with our assignment that the upper diagonal peak arises from GSB, whereas the lower diagonal peak arises from excited state coherences. 


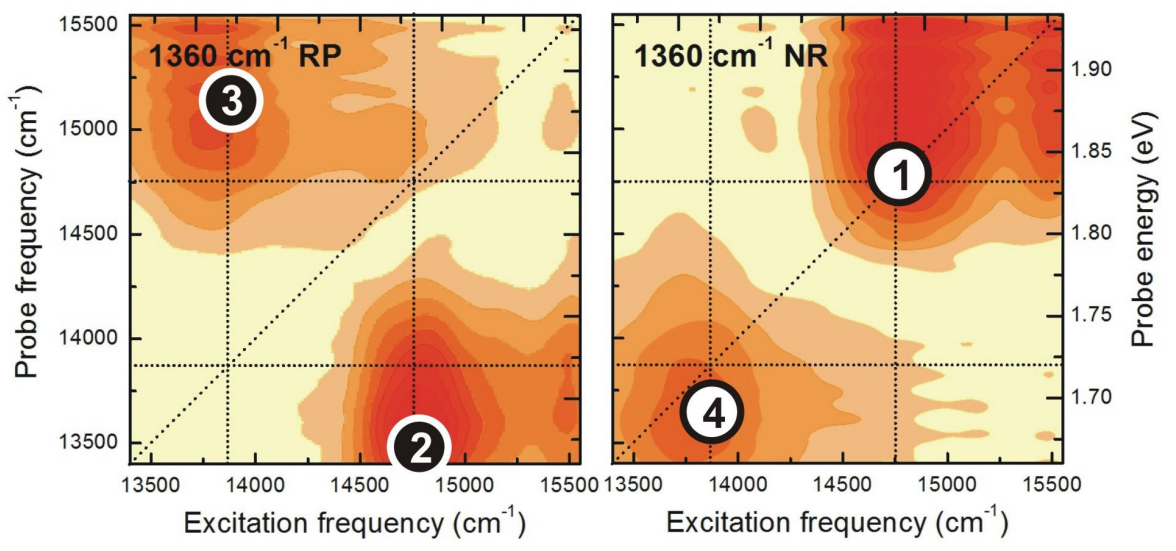

Supplementary Figure S8: Rephasing (left) and non-rephasing (right) beating maps at $1360 \mathrm{~cm}^{-1}$.
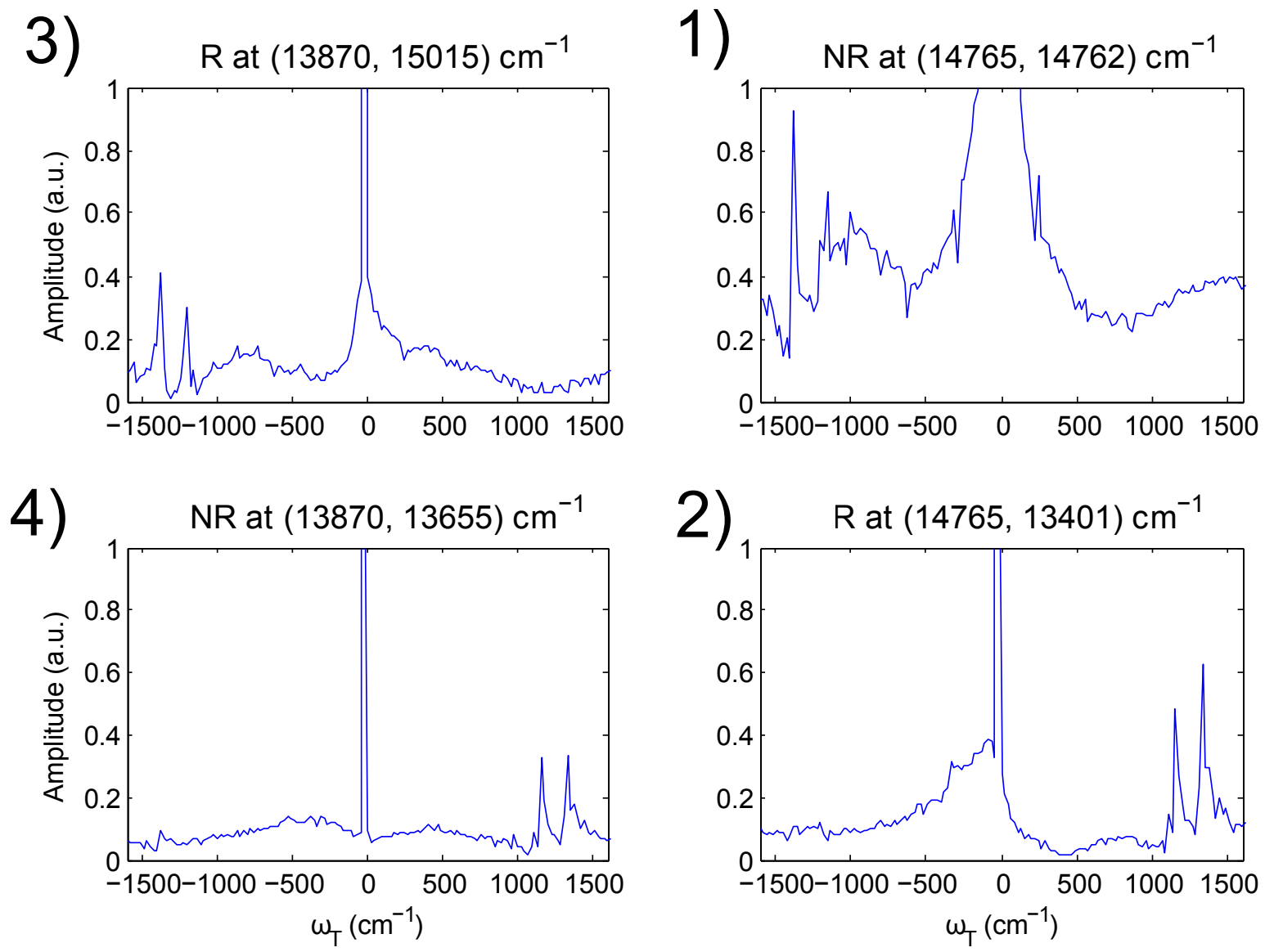

Supplementary Figure S9: Fourier transforms of the complex valued signals at the four points in the 2D spectrum labelled in Supplementary Fig. S8. 
3)

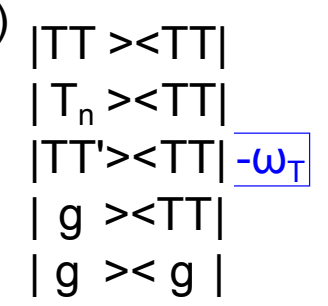

ESA

( $R$ upper cross-peak)

4)

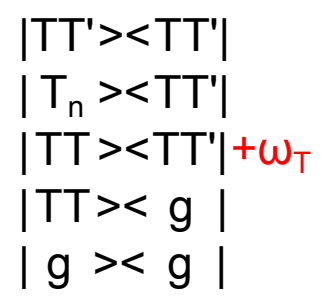

ESA

(NR lower diagonal peak)
1)

$$
\begin{aligned}
& |g><g| \\
& |S><g| \\
& \left|g^{\prime}><g\right|-\omega_{T} \\
& |S><g| \\
& |g><g|
\end{aligned}
$$

GSB

(NR upper diagonal peak)

2)

$$
\begin{aligned}
& \left|g^{\prime}><g^{\prime}\right| \\
& \left|S><g^{\prime}\right| \\
& \left|g><g^{\prime}\right|+\omega_{T} \\
& |g><S| \\
& |g><g|
\end{aligned}
$$

GSB

(R lower cross-peak)

Supplementary Figure S10: Feynman diagrams corresponding to the peaks labelled in Supplementary Fig. S8. The upper diagonal peak and upper cross-peak oscillate at $-\omega_{T}$, whilst the lower diagonal peak and lower cross-peak oscillate at $+\omega_{T}$, as observed in Supplementary Fig. S9.

\section{S.2 Experiment}

\section{S.2.1 2D spectroscopy setup}

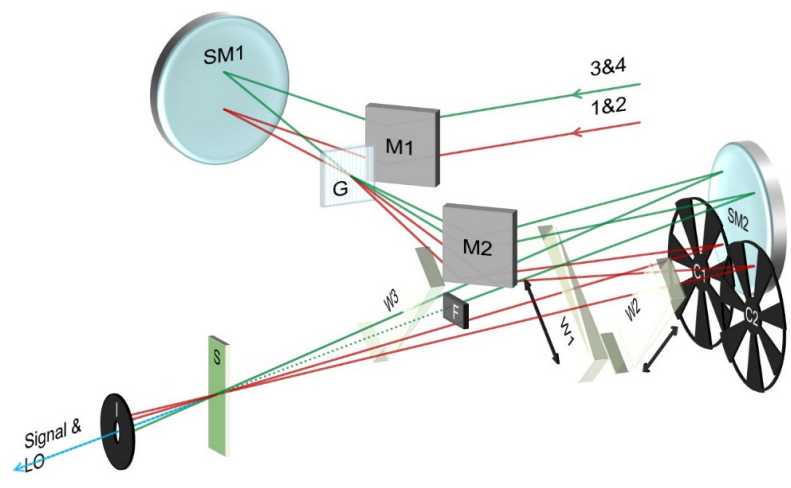

Supplementary Figure S11: The layout of 2D spectroscopy setup with a passive phase stabilisation. 


\section{S.2.2 Absorptive 2D spectra of TIPS}
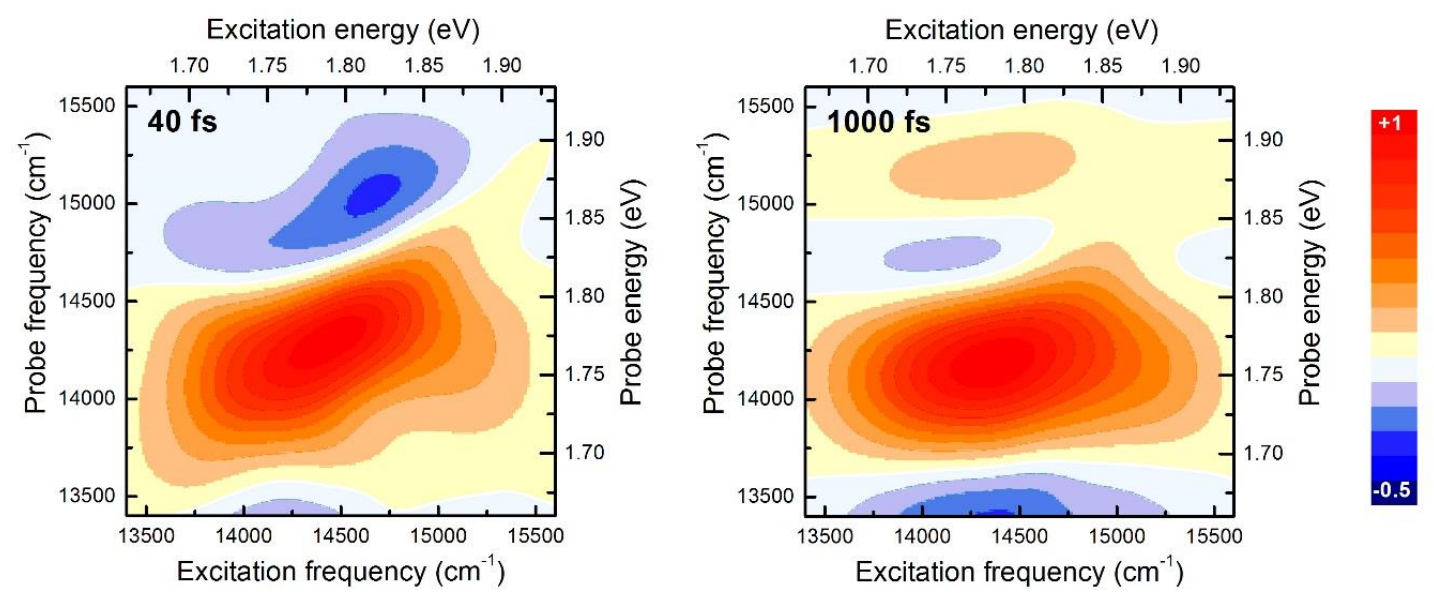

Supplementary Figure S12: 2DES real-part spectra of TIPS molecular crystal at different evolution times.

\section{S.2.3 Absorptive 2D spectra of DTP}
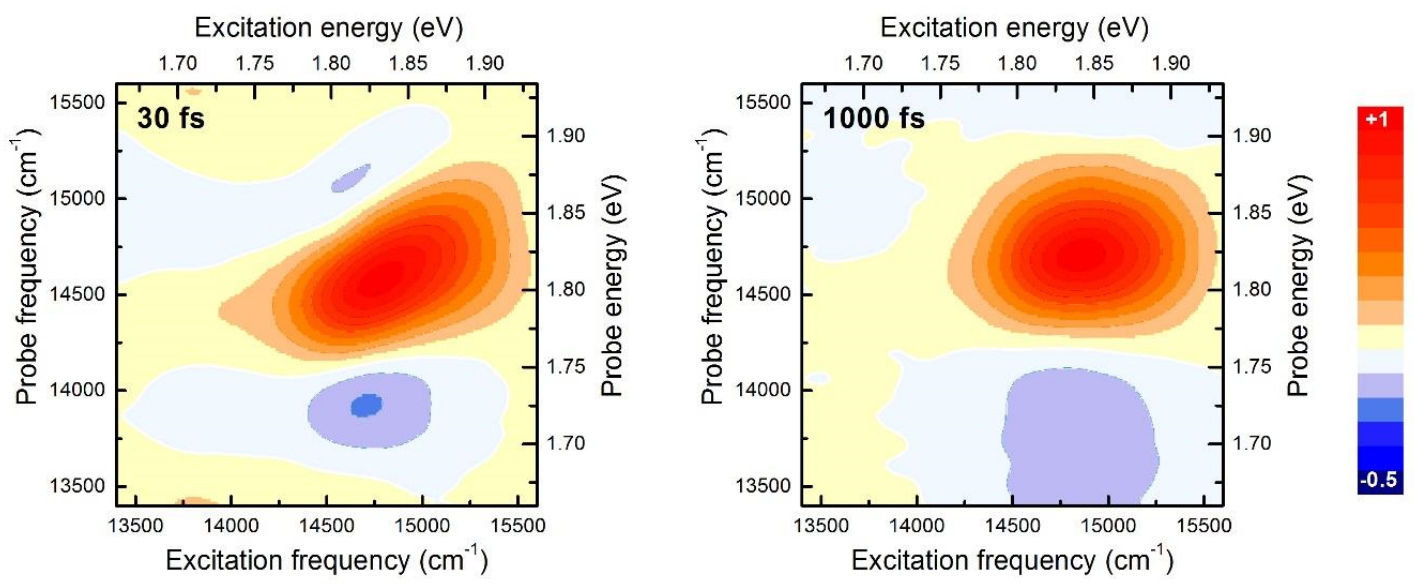

Supplementary Figure S13: 2DES real-part spectra of DTP molecular crystal at different evolution times. 


\section{S.2.4 2D decay associated spectra of pentacene}
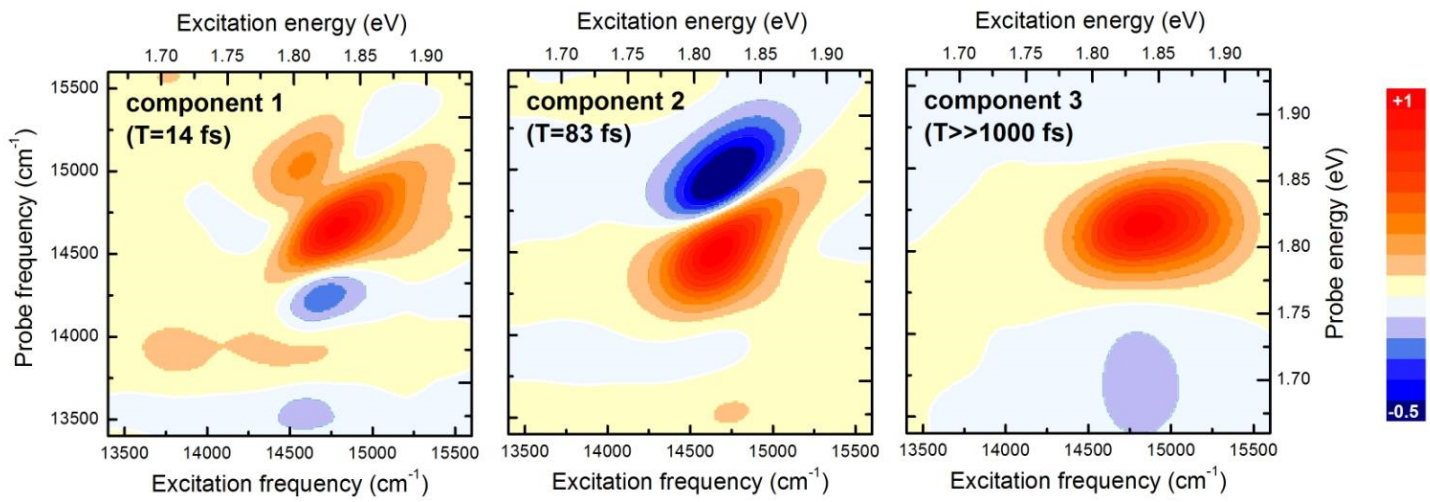

Supplementary Figure S14: 2D decay associated spectra extracted from the real part of 2DES signal for pentacene molecular crystal. 


\section{S.2.5 Beating maps for TIPS and DTP}
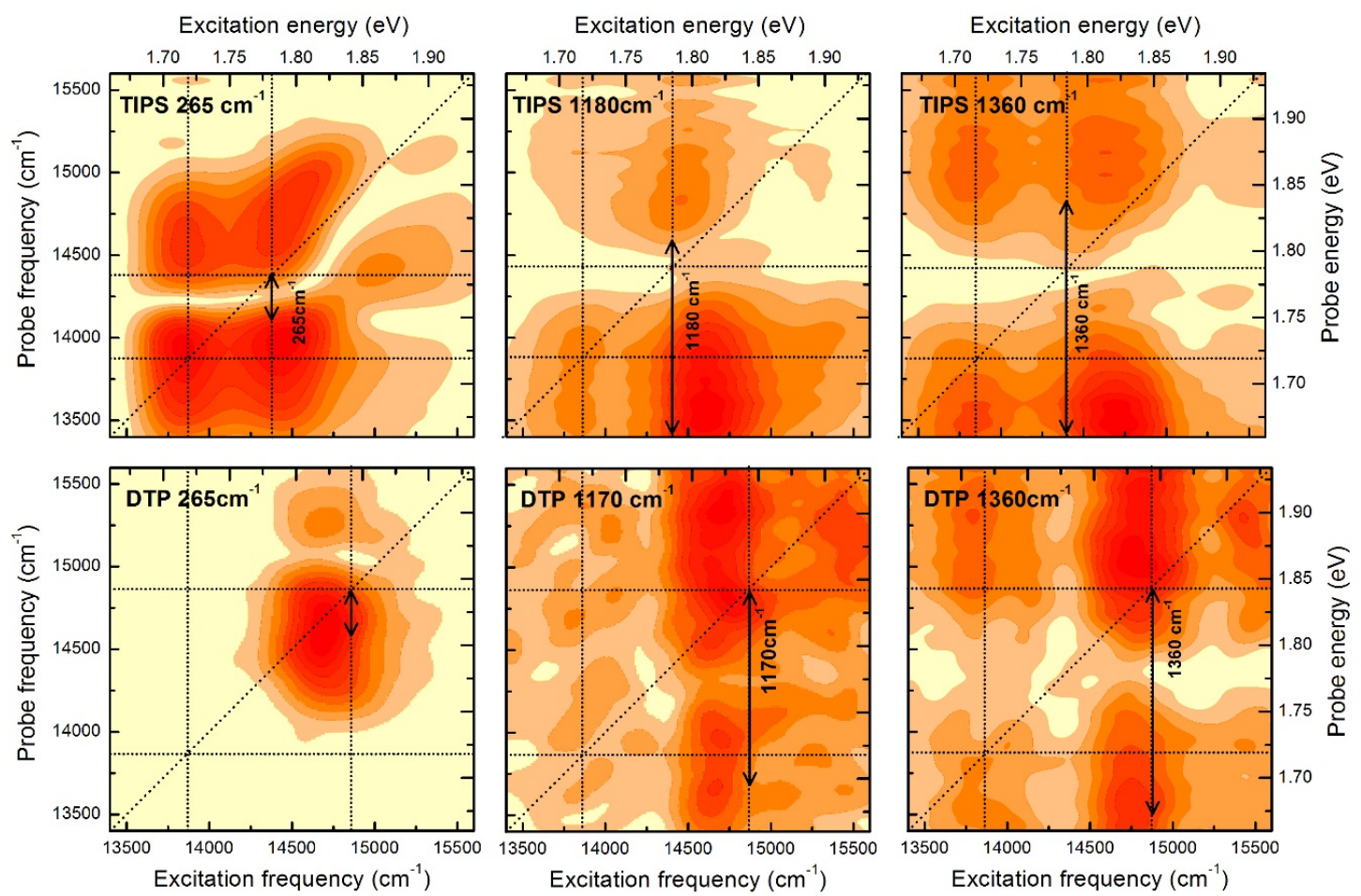

Supplementary Figure S15: The 2D maps of beating in 2DES spectra corresponding to the strongest oscillatory features for TIPS and DTP molecular crystals.

\section{S.2.6 Refractive wings of peaks in $2 \mathrm{D}$ spectra}

In Supplementary Fig. S16 we plot the real rephasing and non-rephasing $2 \mathrm{D}$ spectra at $\mathrm{T}=30$ fs and $\mathrm{T}=1000 \mathrm{fs}$. We observe negative refractive wings in the spectra, which shift from the diagonal in the non-rephasing spectra to off-diagonal positions in the rephasing spectra, as expected[26].

Importantly, these refractive wings are at different positions from the main peaks observed in the beating maps; in particular there is no overlap with the peaks observed with excitation at the energy of the multiexcitonic state. Moreover, if there was any overlap with the oscillating signals observed with excitation at the energy of the multiexcitonic state, we would observe oscillations with negative frequency in part 4 of Supplementary Fig. S9, which we do not.

The only place where the refractive wings can contribute significantly to the beating maps is in the smaller peaks observed around peak 1 in Supplementary Fig. S7. Indeed the peaks observed at $\left(\omega_{\tau}, \omega_{t}\right)=(14500,15000) \mathrm{cm}^{-1}$ and $(14750,15250) \mathrm{cm}^{-1}$ in the experimental rephasing and non-rephasing $265 \mathrm{~cm}^{-1}$ beating maps respectively may come from these wings. Note that our simulations of the beating maps utilize a Lorentzian lineshape for the peaks, which accounts for the effects of the negative refractive wings. 

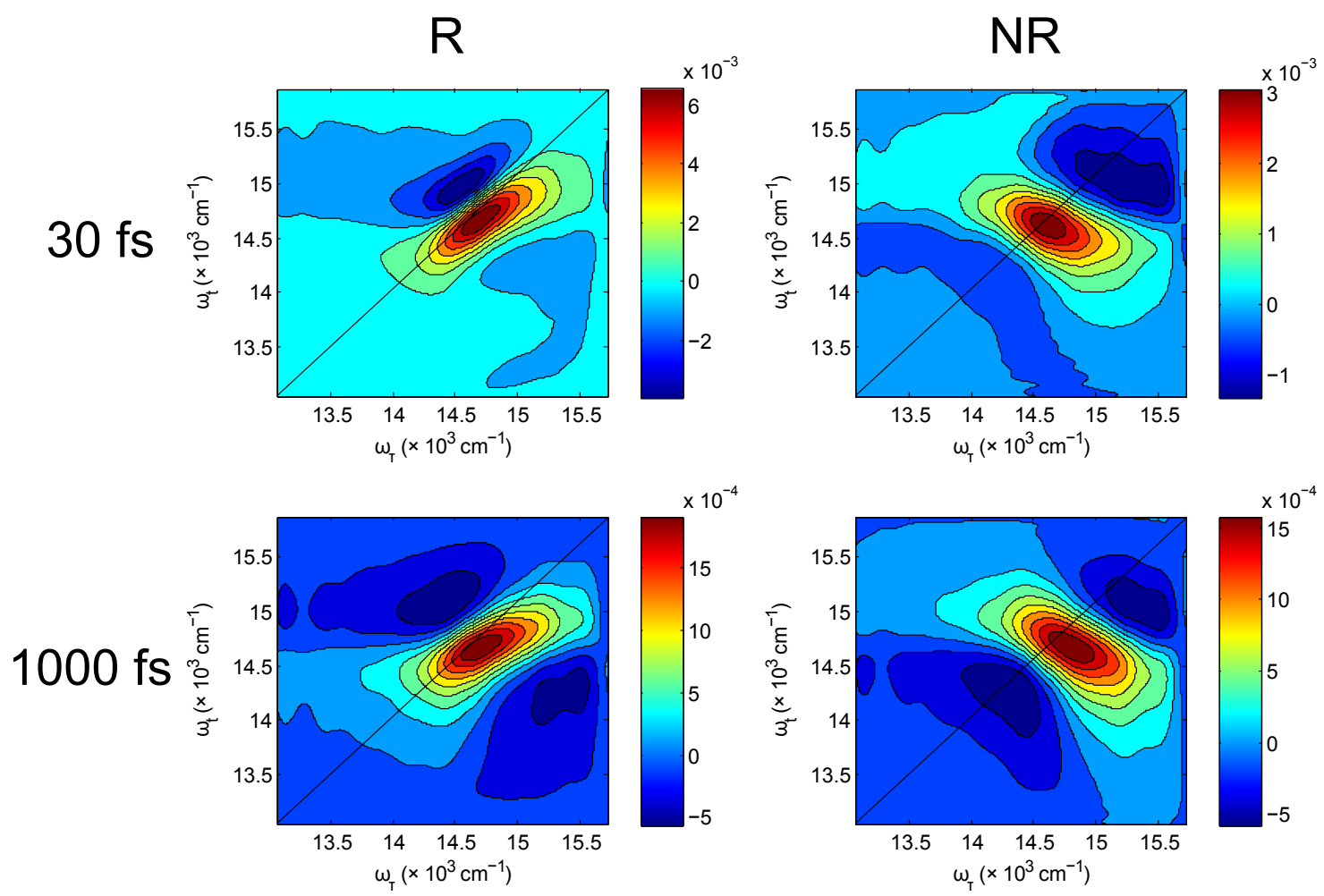

Supplementary Figure S16: Real rephasing (left) and non-rephasing (right) 2D experimental spectra at $\mathrm{T}=30$ fs (top) and $\mathrm{T}=1000 \mathrm{fs}$ (bottom). 


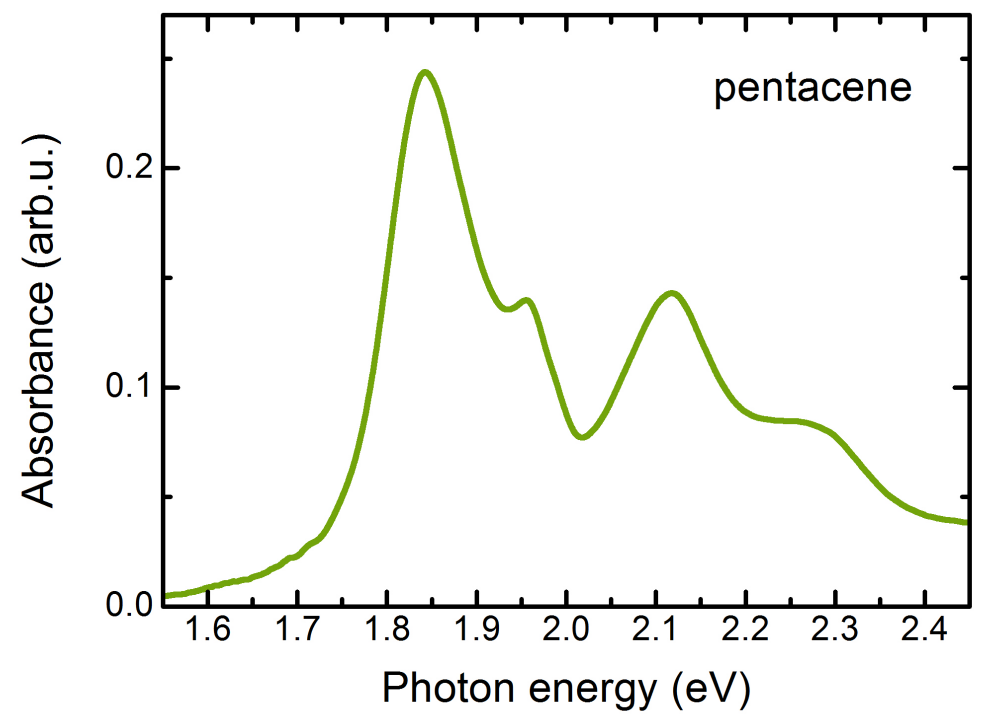

Supplementary Figure S17: Absorption spectrum of the studied pentacene film in a wide photon-energy range.

\section{S.2.7 2D response at high photon energy excitation/probe (from 2 to $2.4 \mathrm{eV}$ )}

To confirm the results and their interpretation obtained in the excitation/probe range of $1.6-1.95 \mathrm{eV}$ we have performed complementary 2D measurements of pentacene films in the region between 2 and 2.4 $\mathrm{eV}$. The absorption spectra of pentacene (Supplementary Figure S17) demonstrates a clear absorption peak associated with a transition to one of the singlet exciton states, which we notate as $\mathrm{S}_{\mathrm{HE}}$. It is also know from previous studies [27] that performing the experiment with a tilted film allows detection of a triplet ESA transition at $2.3 \mathrm{eV}$, which opens the opportunity to track the fission process and potentially resolve the vibronic coherence in the multiexciton state.

Supplementary Figure S18 presents 2D absorptive spectra of 60-degree tilted [27] pentacene film at 80 fs and $900 \mathrm{fs}$ evolution times. While time resolution of the experiment was $\sim 12 \mathrm{fs}$, the $2 \mathrm{D}$ spectra at evolution times below 80 fs were contaminated by a strong scattering signal and excluded from analysis. At early evolution times, the response is dominated by the GSB signals coming from the main absorption peak at $2.15 \mathrm{eV}$ and a smaller absorption peak at $2.26 \mathrm{eV}$. This GSB signal is steady in time as seen in the kinetics presented in Supplementary Fig. S19. This is expected, as both singlet and triplet excitons are bleaching the ground state to singlet transition and the GSB response should persist over the entire lifetime of the photoexcited species. In contrast to the $2 \mathrm{D}$ data at the lowest singlet energy $(1.83 \mathrm{eV})$ no SE contribution to the observed spectra can be found, as the high energy singlet states have many extra ultrafast relaxation channels, in addition to singlet fission, and this signal vanishes within our time resolution. A slight $\sim 300 \mathrm{fs}$ increase is likely to be associated with a minor heating contribution[28]. At the same time, a strong ESA signal builds up at a rate of 90 fs in the $2.30 \mathrm{eV}$ region (Supplementary Figures S18 and S19). Based on previous studies[27], we associate this signal with the transition to a higher-lying triplet state.

These new data are in perfect agreement with previous studies as well as with the measurements in the $1.6-1.95 \mathrm{eV}$ range. They confirm that fission occurs on the timescale of 90(87) fs and that the long-lived ESA features observed at $2.3 \mathrm{eV}, 1.9 \mathrm{eV}$, and $1.7 \mathrm{eV}$ all correspond to the transitions between the triplet excited states. 

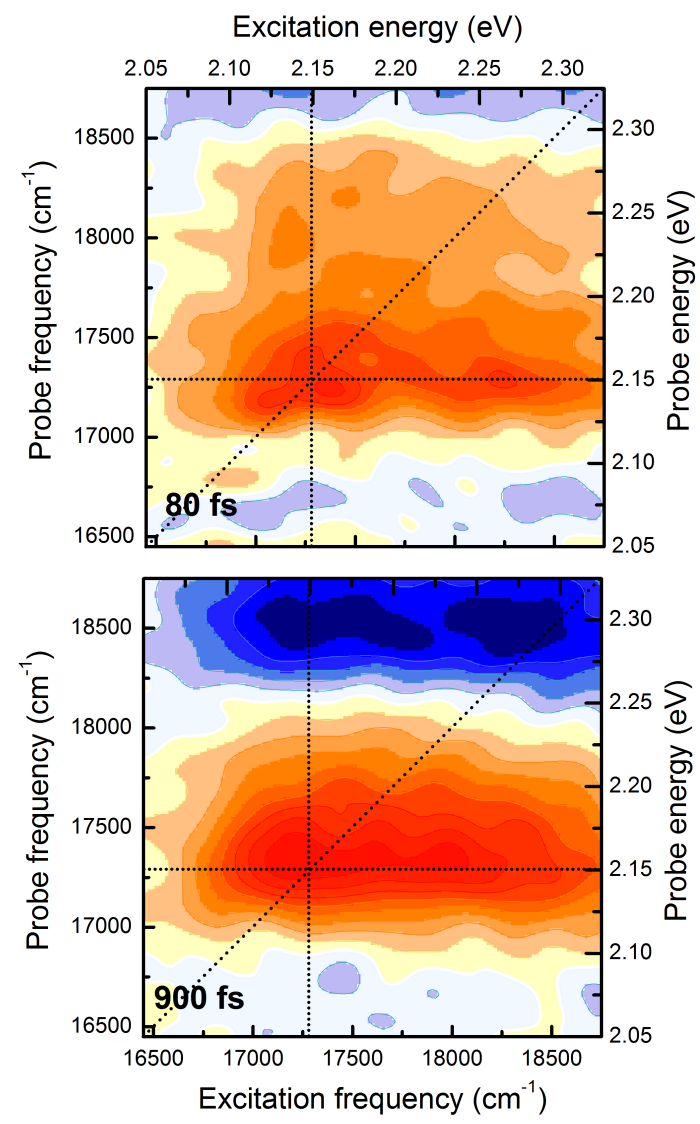

Supplementary Figure S18: 2DEPE real valued spectra of pentacene film at different evolution times. Red stands for bleaching and blue for absorption signals. 


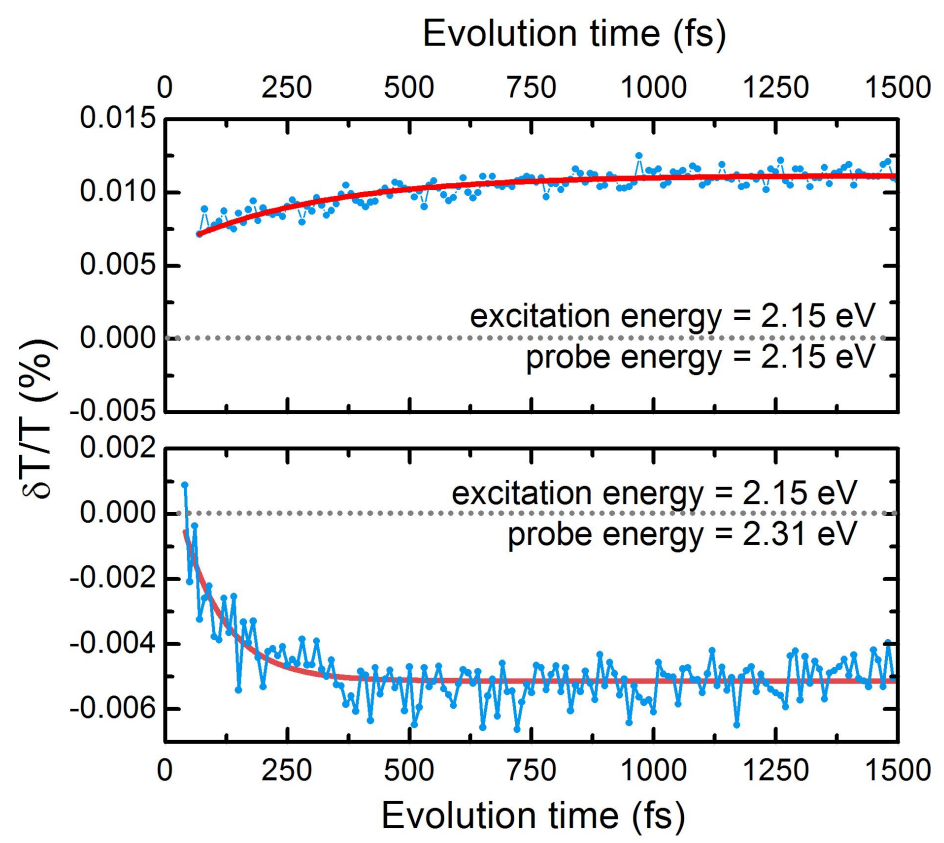

Supplementary Figure S19: Evolution-time transients corresponding to the different locations in 2D spectrum of pentacene. Red curves are the exponential fits (time constants: top $300 \mathrm{fs}$, bottom $90 \mathrm{fs}$ ).

\section{S.2.8 Beating maps for the high photon energy excitation data}

Here we discuss the evolution time dynamics for the data obtained at high photon excitation energy. Due to high scattering in this energy region, the quality of the data is worse than the data obtained at lower energies, however certain trends can still be observed.

We plot the integrated spectrum of evolution time oscillations observed in the data in Supplementary Fig. S20. As before, we observe oscillations at 265,1170 and $1360 \mathrm{~cm}^{-1}$. The oscillations at 1170 and $1360 \mathrm{~cm}^{-1}$ are the strongest and we plot the corresponding beating maps in Supplementary Fig. S21.

In each beating map, we observe single peaks with excitation energies corresponding to the energy of the $\mathrm{S}_{0}$ to $\mathrm{S}_{\mathrm{HE}}$ transition, but which have lower emission energies in the non-rephasing beating maps than in the rephasing beating maps. The difference between the emission energies roughly matches the energy of the vibration (i.e. 1170 or $1360 \mathrm{~cm}^{-1}$ ). This change in emission frequency between the rephasing and nonrephasing maps indicates that the oscillations arise from vibrational coherences in an excited state during the evolution time. The opposite pattern is expected for ground state bleach signals, see discussion in Section S.1.6 for more details (the different peak pattern expected for ground state bleach also been discussed in the literature, see for example [22, 29]). The peak pattern resembles that of peaks 3 and 4 in Figure $5 \mathrm{a}$ of the main text.

To further test this assignment, in Supplementary Fig. S22 we inspect the sign of the Fourier transforms taken at specific points within the peaks of the beating maps. The sign of the oscillations is negative in the rephasing spectrum peak and positive in the non-rephasing spectrum peak, which rules out the possibility of ground state bleach (see Section S.1.6). Therefore we conclude that we observe excited state coherences during the evolution time. Moreover, the probe energy we observe is around the energy of known triplet ESA transitions[27] (at about $18500 \mathrm{~cm}^{-1}$, leading to emission at $18500 \mathrm{~cm}^{-1}$ in the rephasing map and $18500 \mathrm{~cm}^{-1}-\omega$ in the non-rephasing map), suggesting that the excited state coherences evolve on the triplet state during the evolution time. The model of Section S.1.2 provides a mechanism for these spectroscopic 


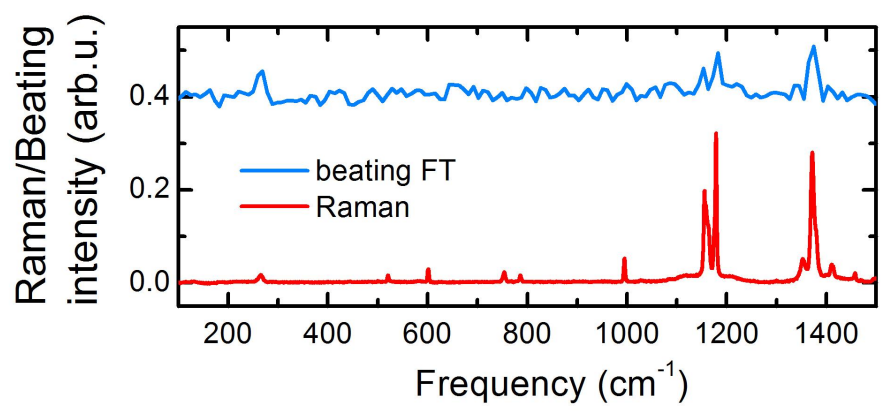

Supplementary Figure S20: Integrated spectrum of evolution time oscillations observed in 2DES high photon energy data.
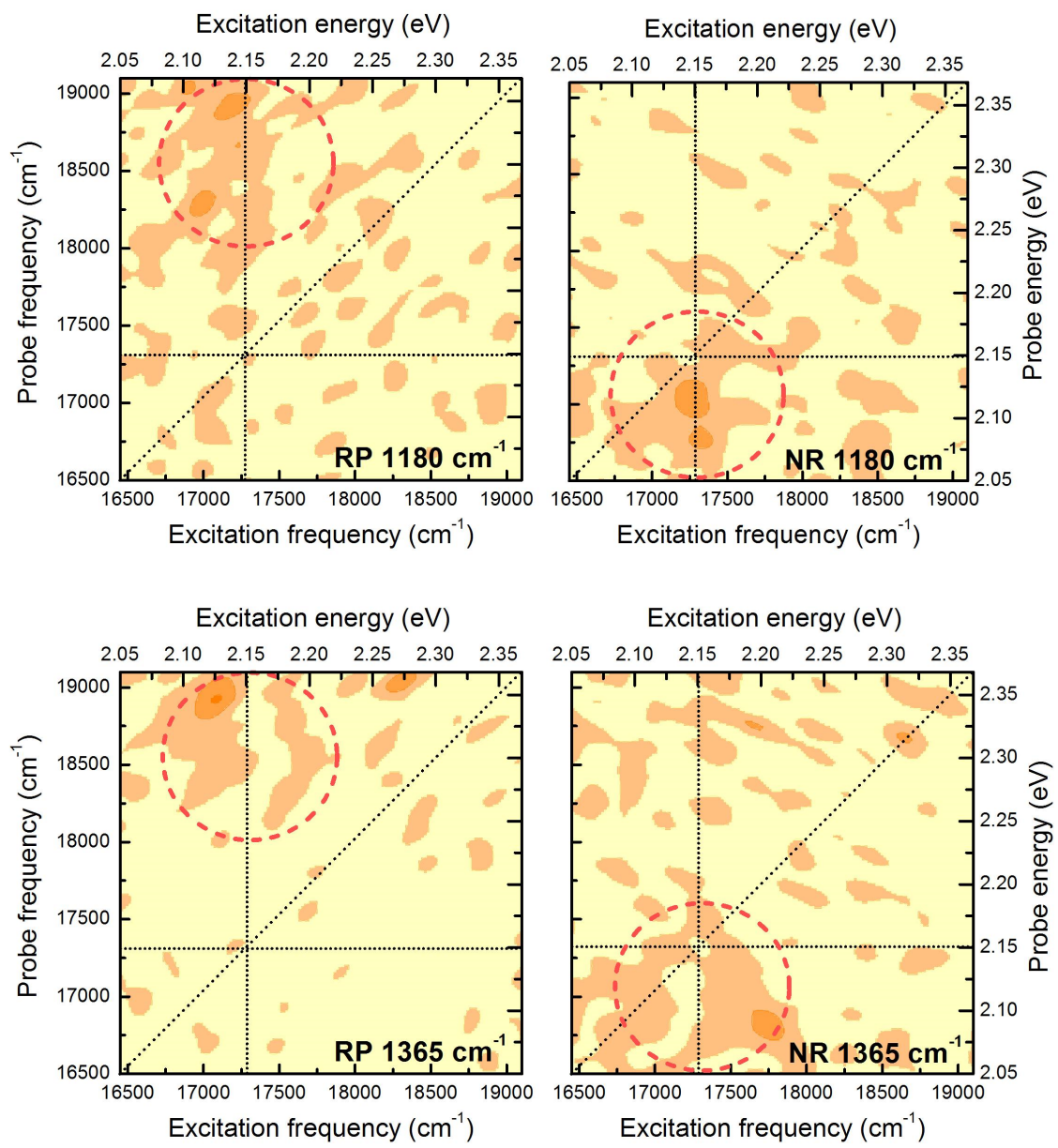

Supplementary Figure S21: Experimental beating maps for the high photon energy excitation data in the regions of $1170 \mathrm{~cm}^{-1}$ and $1360 \mathrm{~cm}^{-1}$. 

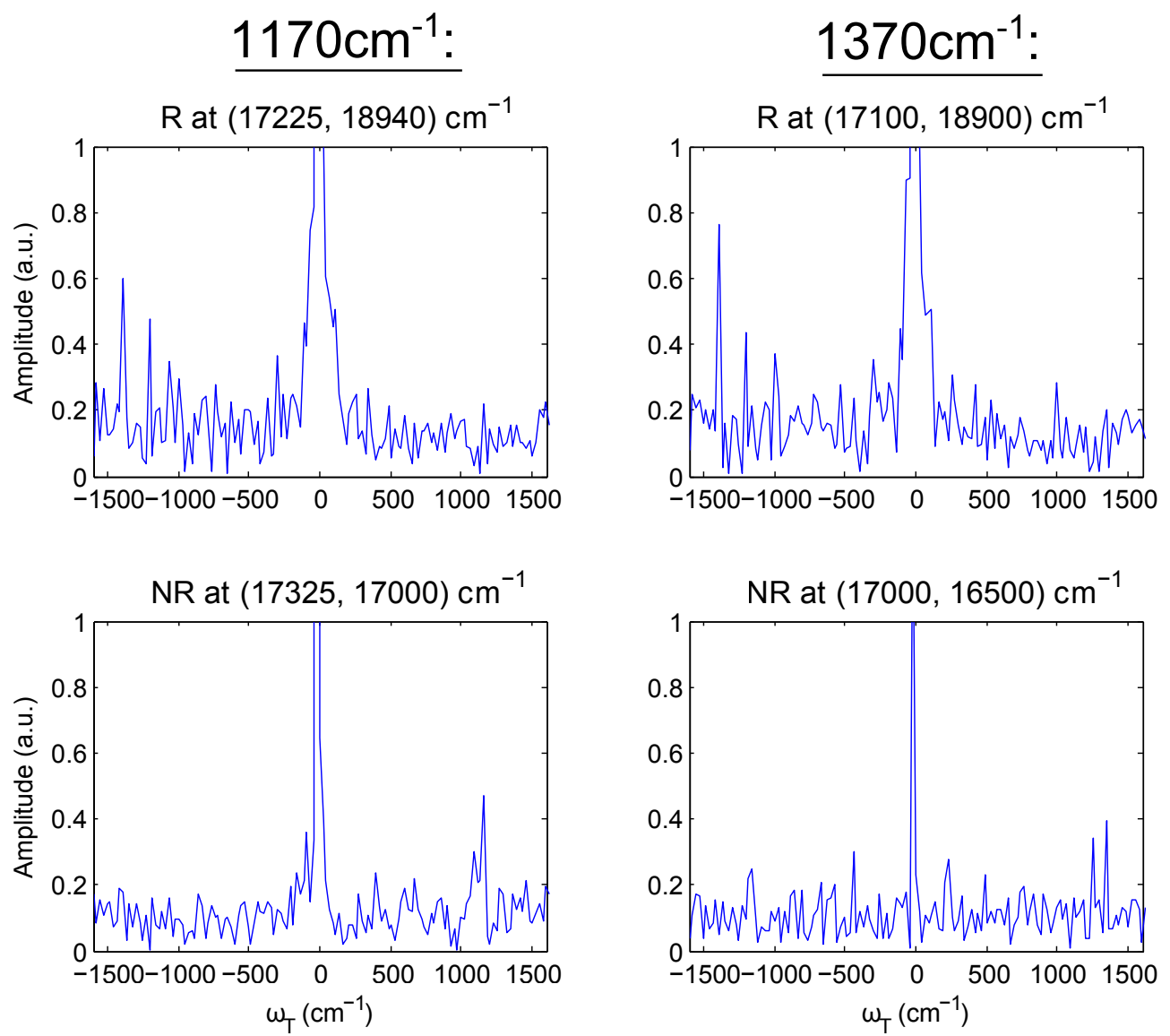

Supplementary Figure S22: Fourier transforms of the high photon energy excitation data over the evolution time at the points indicated in the $2 \mathrm{D}$ spectra.

signatures of triplet coherence, which we also observed and explained in Section S.1.4.

\section{S.2.9 Cross-polarisation data}

In order to further test our model, we performed additional 2D spectroscopy experiments using a crosspolarisation sequence, where the polarisation orientation of pulses one to four was chosen to be $\pi / 4,-\pi / 4$, $\pi / 2$ and 0 respectively (this is in contrast to the all parallel polarisation pulse sequence employed throughout the rest of this work). This cross-polarisation sequence only allows signals to be observed if the transition dipole moments of the states excited by the first and second pulses have different orientations [30]. This means that signals of purely vibrational origins are suppressed. This technique comes from 2D IR spectroscopy [31] and has already been used to distinguish signals with electronic and vibrational origins in 2D optical spectroscopy; see for example [30, 32].

We plot the integrated spectrum of evolution time oscillations observed in the data in Supplementary Fig. S23. Notice that we obtain two high frequency peaks, at around 1170 and $1360 \mathrm{~cm}^{-1}$, as observed in the data with all-parallel pulse polarisations. However, we no longer observe a peak at $265 \mathrm{~cm}^{-1}$. This suggests that the signals observed in the $265 \mathrm{~cm}^{-1}$ beating map are purely vibrational (mainly on the ground state) in origin and are therefore suppressed by the cross-polarisation sequence, whilst the signals observed in 


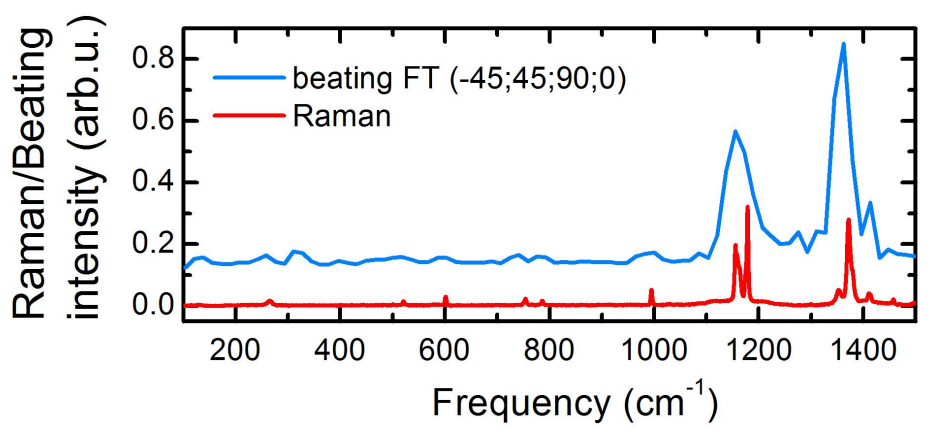

Supplementary Figure S23: Integrated spectrum of evolution time oscillations observed in 2DES crosspolarisation data.

the higher frequency beating maps have mixed vibrational and electronic origins (in the excited state). This supports our proposed model, as vibrational excitations of the $265 \mathrm{~cm}^{-1}$ mode on the mutliexcitonic state are far from resonance states with the singlet exciton and cannot effectively participate in vibronic singlet-multiexciton state mixing.

The corresponding high frequency beating maps are plotted in Supplementary Fig. S24. We observe similar peak patterns to those in Figure 5a of the main text. The peaks which absorb at $\omega_{\tau} \approx 1.72 \mathrm{eV}$ (the energy of the multiexciton state, TT) are known to arise from ESA and evolve in a vibrational coherence on the multiexciton TT state during the evolution time (see discussion in Sections S.1.5 and S.1.6 above). The fact that these signals survive the polarisation sequence suggests that the multiexciton state with no vibrational excitations (TT) and the vibrationally excited multiexciton state (TT') have transition dipole moments with different orientations due to different amounts of mixing with the singlet state. This supports our model that the high frequency vibrations allow for mixing between the singlet and multiexciton states. 

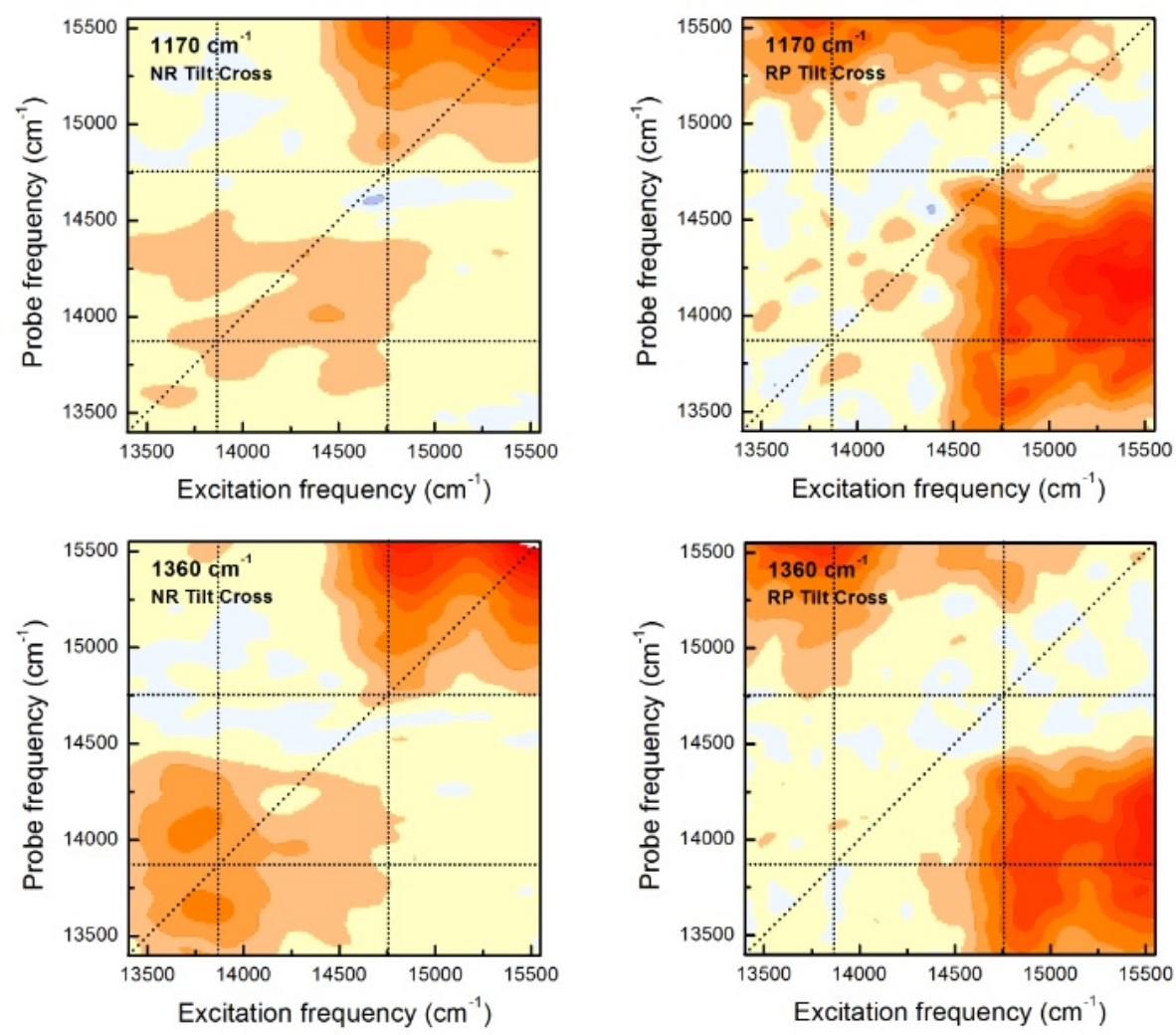

Supplementary Figure S24: The 2D beating maps corresponding to the strongest oscillatory features observed in Supplementary Fig. S23 (with cross-polarisation sequence). 


\section{References}

[1] T. C. Berkelbach, M. S. Hybertsen, and D. R. Reichman, "Microscopic theory of singlet exciton fission. II. Application to pentacene dimers and the role of superexchange," J. Chem. Phys., vol. 138, no. 11, p. 114103, 2013.

[2] T. C. Berkelbach, M. S. Hybertsen, and D. R. Reichman, "Microscopic theory of singlet exciton fission. III. Crystalline pentacene," J. Chem. Phys., vol. 141, p. 074705, 2014.

[3] M. B. Smith and J. Michl, "Recent advances in singlet fission," Annual Review of Physical Chemistry, vol. 64, pp. 361-386, 2013.

[4] X. Feng, A. V. Luzanov, and A. I. Krylov, "Fission of entangled spins: An electronic structure perspective," J. Phys. Chem. Lett., vol. 4, pp. 3845-3852, 2013.

[5] T. Zeng, R. Hoffmann, and N. Ananth, "The low-lying electronic states of pentacene and their roles in singlet fission," J. Am. Chem. Soc., vol. 136, pp. 5755-5764, 2014.

[6] P. Coto, S. Sharifzadech, J. B. Neaton, and M. Thoss, "Low-lying electronic excited states of pentacene oligomers: A comparative electronic structure study in the context of singlet fission," J. Chem. Theory Comput., 2015.

[7] D. Beljonne, H. Yamagata, J. Brédas, F. Spano, and Y. Olivier, "Charge-transfer excitations steer the Davydov splitting and mediate singlet exciton fission in pentacene," Physical Review Letters, vol. 110, no. 22, p. 226402, 2013.

[8] T. C. Berkelbach, M. S. Hybertsen, and D. R. Reichman, "Microscopic theory of singlet exciton fission. I. General formulation," J. Chem. Phys., vol. 138, no. 11, p. 114102, 2013.

[9] W.-L. Chan, M. Ligges, A. Jailaubekov, L. Kaake, L. Miaja-Avila, and X.-Y. Zhu, "Observing the multiexciton state in singlet fission and ensuing ultrafast multielectron transfer," Science, vol. 334, no. 6062, pp. 1541-1545, 2011.

[10] W.-L. Chan, T. C. Berkelbach, M. R. Provorse, N. R. Monahan, J. R. Tritsch, M. S. Hybertsen, D. R. Reichman, J. Gao, and X.-Y. Zhu, "The quantum coherent mechanism for singlet fission: Experiment and theory," Accounts of Chemical Research, vol. 46, no. 6, pp. 1321-1329, 2013.

[11] J. C. Johnson, A. J. Nozik, and J. Michl, "The role of chromophore coupling in singlet fission," Accounts of chemical research, vol. 46, no. 6, pp. 1290-1299, 2013.

[12] M. B. Smith and J. Michl, "Singlet fission," Chemical Reviews, vol. 110, no. 11, pp. 6891-6936, 2010.

[13] P. M. Zimmerman, Z. Zhang, and C. B. Musgrave, "Singlet fission in pentacene through multi-exciton quantum states," Nature Chemistry, vol. 2, no. 8, pp. 648-652, 2010.

[14] P. M. Zimmerman, F. Bell, D. Casanova, and M. Head-Gordon, "Mechanism for singlet fission in pentacene and tetracene: From single exciton to two triplets," Journal of the American Chemical Society, vol. 133, no. 49, pp. 19944-19952, 2011.

[15] P. M. Zimmerman, C. B. Musgrave, and M. Head-Gordon, "A correlated electron view of singlet fission," Accounts of Chemical Research, vol. 46, no. 6, pp. 1339-1347, 2013.

[16] W.-L. Chan, M. Ligges, and X. Zhu, "The energy barrier in singlet fission can be overcome through coherent coupling and entropic gain," Nature Chemistry, vol. 4, no. 10, pp. 840-845, 2012.

[17] A. Rao, M. W. Wilson, J. M. Hodgkiss, S. Albert-Seifried, H. Bässler, and R. H. Friend, "Exciton fission and charge generation via triplet excitons in pentacene/C60 bilayers," Journal of the American Chemical Society, vol. 132, no. 36, pp. 12698-12703, 2010.

[18] F. Anger, J. Ossó, U. Heinemeyer, K. Broch, R. Scholz, A. Gerlach, and F. Schreiber, "Photoluminescence spectroscopy of pure pentacene, perfluoropentacene, and mixed thin films," J. Chem. Phys., vol. 136, no. 5, p. 054701, 2012. 
[19] L. Gisslen, "Influence of Frenkel excitons and charge transfer states on the spectroscopic properties of organic molecular cystrals," Ph. D. Thesis, Technische Universitat Munchen, 2009.

[20] D. Egorova, M. Thoss, W. Domcke, and H. Wang, "Modeling of ultrafast electron-transfer processes: Validity of multilevel redfield theory," J. Chem. Phys., vol. 119, no. 5, pp. 2761-2773, 2003.

[21] S. Mukamel, Principles of Nonlinear Optical Spectroscopy, Oxford Series on Optical and Imaging Sciences. Oxford University Press, 1999.

[22] D. Egorova, "Self-analysis of coherent oscillations in time-resolved optical signals," The Journal of Physical Chemistry A, vol. 118, no. 44, pp. 10259-10267, 2014.

[23] J. Seibt and T. Pullerits, "Beating signals in 2D spectroscopy: Electronic or nuclear coherences? application to a quantum dot model system," J. Phys. Chem. C, vol. 117, pp. 18728-18737, 2013.

[24] H. Li, A. D. Bristow, M. E. Siemens, G. Moody, and S. T. Cundiff, "Unraveling quantum pathways using optical 3D Fourier-transform spectroscopy," Nature Communications, vol. 4, p. 1390, 2013.

[25] Y. Song, C. Hellmann, N. Stingelin, and G. D. Scholes, "The separation of vibrational coherence from ground- and excited-electronic states in P3HT film," J. Chem. Phys., vol. 142, p. 212410, 2015.

[26] M. Cho, Two-Dimensional Optical Spectroscopy. CRC Press, Taylor and Francis Group, 2009.

[27] M. Wilson, A. Rao, B. Ehrler, and R. Friend, "Singlet exciton fission in polycrystalline pentacene: From photophysics toward devices," Acc. Chem. Res., vol. 46, p. 1330, 2013.

[28] A. Rao, M. W. Wilson, S. Albert-Seifried, R. Di Pietro, and R. H. Friend, "Photophysics of pentacene thin films: The role of exciton fission and heating effects," Physical Review B, vol. 84, no. 19, p. 195411, 2011.

[29] V. Butkus, D. Zigmantas, L. Valkunas, and D. Abramavicius, "Vibrational vs. electronic coherences in 2D spectrum of molecular systems," Chemical Physics Letters, vol. 545, pp. 40-43, 2012.

[30] G. Schlau-Cohen, A. Ishizaki, T. Calhoun, N. Ginsberg, M. Ballottari, R. Bassi, and G. Fleming, "Elucidation of the timescales and origins of quantum electronic coherence in LHCII," Nature Chemistry, vol. 4, p. 389, 2012.

[31] M. Zanni, N.-H. Ge, Y. S. Kim, and R. Hochstrasser, "Two-dimensional IR spectroscopy can be designed to eliminate the diagonal peaks and expose only the crosspeaks needed for structure determination," PNAS, vol. 98, p. 11265, 2001.

[32] S. Westenhoff, D. Palec̃ek, P. Edlund, P. Smith, and D. Zigmantas, "Coherent picosecond exciton dynamics in a photosynthetic reaction center," JACS, vol. 134, p. 16484, 2012. 